\title{
Efficient implementation of Galerkin meshfree methods for large-scale problems with an emphasis on maximum entropy approximants
}

\author{
Christian Peco, Daniel Millán, Adrian Rosolen and Marino Arroyo* \\ LaCàN, Universitat Politècnica de Catalunya (UPC), Barcelona 08034, Spain
}

\begin{abstract}
In Galerkin meshfree methods, because of a denser and unstructured connectivity, the creation and assembly of sparse matrices is expensive. Additionally, the cost of computing basis functions can be significant in problems requiring repetitive evaluations. We show that it is possible to overcome these two bottlenecks resorting to simple and effective algorithms. First, we create and fill the matrix by coarse-graining the connectivity between quadrature points and nodes. Second, we store only partial information about the basis functions, striking a balance between storage and computation. We show the performance of these strategies in relevant problems.
\end{abstract}

Keywords: meshfree methods, local maximum entropy, sparse matrix efficient assembly, matrix structure creation, optimal memory storage, code optimization

\section{Introduction}

Meshfree methods have emerged in recent years as a viable alternative to finite elements in a number of applications, see $[1,2,3,4,5]$ for a detailed review. These methods are based on basis functions that do not rely on a mesh. As a consequence, many of the requirements associated with the quality of the elements in traditional finite element method (FEM) are relaxed or disappear, but this extra flexibility raises new challenges in the numerical implementation [6]. Meshfree methods also present several advantages such as basis functions with high-order continuity, robustness in dramatic grid deformations [7, 8, 9], and easier local adaptivity [10, 11]. Galerkin meshfree methods require a quadrature mesh to perform numerical integration, commonly requiring a higher number of quadrature points to accurately integrate the weak form due to their nonpolynomial nature and nonelement-wise support [12,13]. Additionally, most of the meshfree methods present an awkward treatment of essential boundary conditions due to nonsatisfaction of the Kronecker delta property [3, 14].

\footnotetext{
*Correspondence to: marino.arroyo@upc.edu
} 


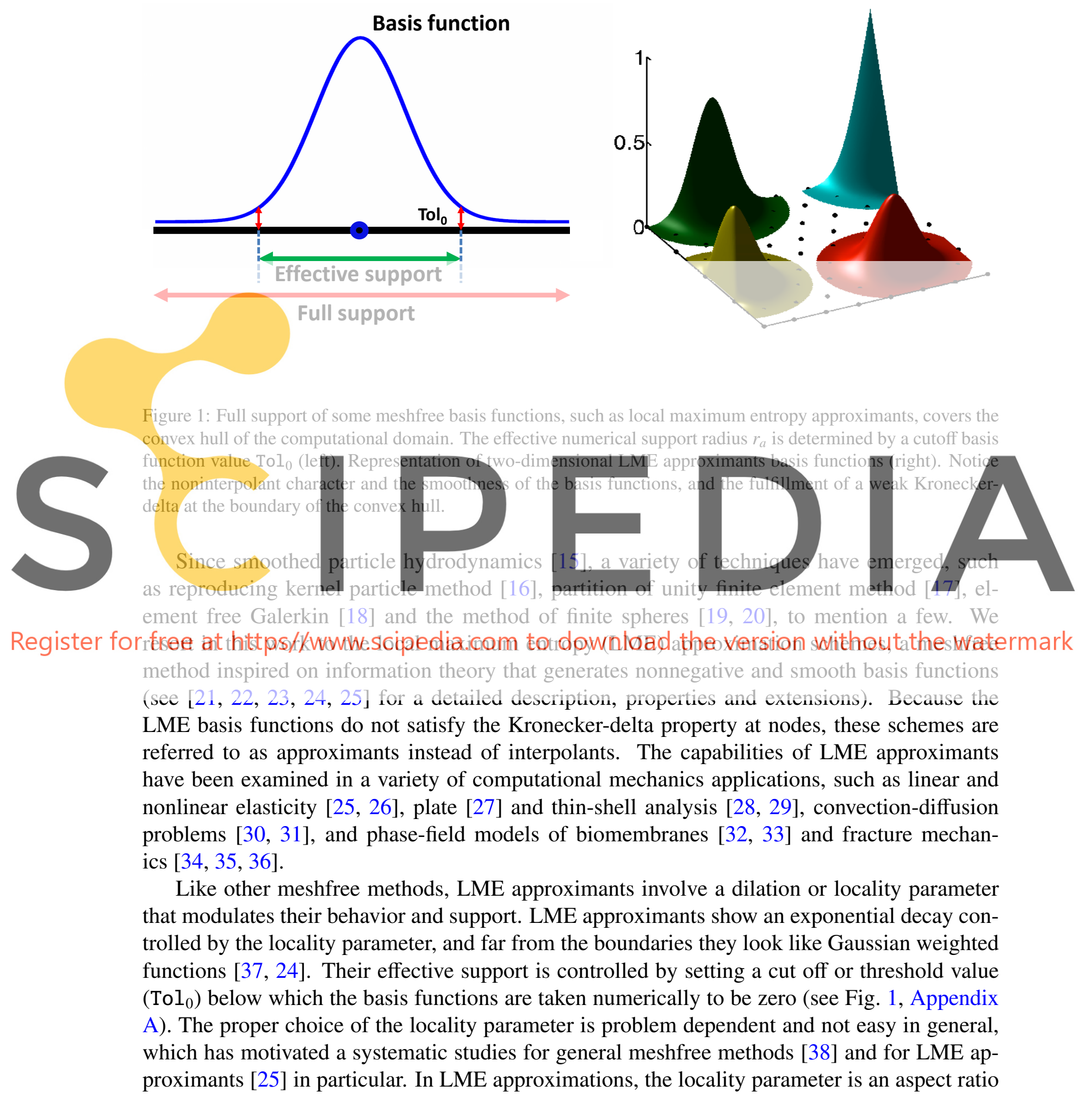


parameter $\gamma$, which allows us to smoothly move from linear finite elements shape functions $(\gamma>4.0)$ to more spread out approximation schemes (e.g., $\gamma=0.6)$, as illustrated in Fig. 2. In general, broader functions lead to more accurate results for problems with smooth solutions at the expense of higher computational cost and worse matrix conditioning [22, 28].

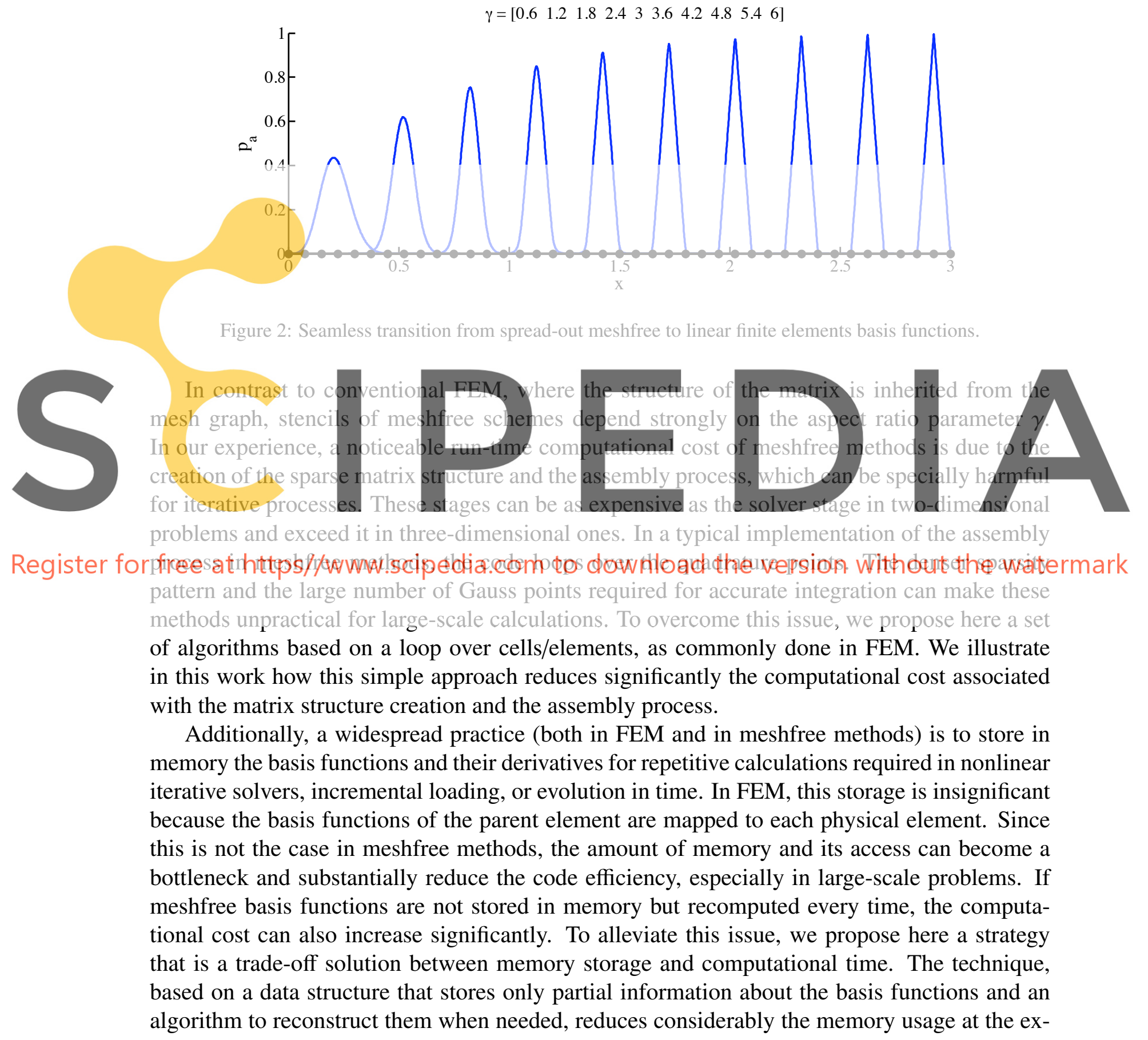


pense of a minimum increment in the overall computational cost. We illustrate and exploit this concept on LME approximants.

The paper is organized as follows. In Section 2 we review the basic technicalities for a meshfree method particularized to LME approximants and the classical implementation to approximate partial differential equations (PDEs). We then propose an algorithm to speed-up the matrix assembly and an algorithm for the compressed memory storage of LME approximants in Section 3. We extensively test our proposals with numerical examples in Section 4 and finish with some concluding remarks in Section 5.

\section{A standard meshfree scheme}

Let $X=\left\{x_{1}, x_{2}, \ldots, x_{N}\right\} \subset \mathbb{R}^{d}$, for $d=1,2,3$, be an unstructured set of nodes used to describe a domain $\Omega$, and $p_{a}(\boldsymbol{x})$ the meshfree basis function associated to the $a$-th node, for $a=1, \ldots, N$. A continuous field $\Phi$ can be approximated as

$$
\Phi(x)=\sum_{a=1}^{N} p_{a}(x) \Phi_{a}
$$
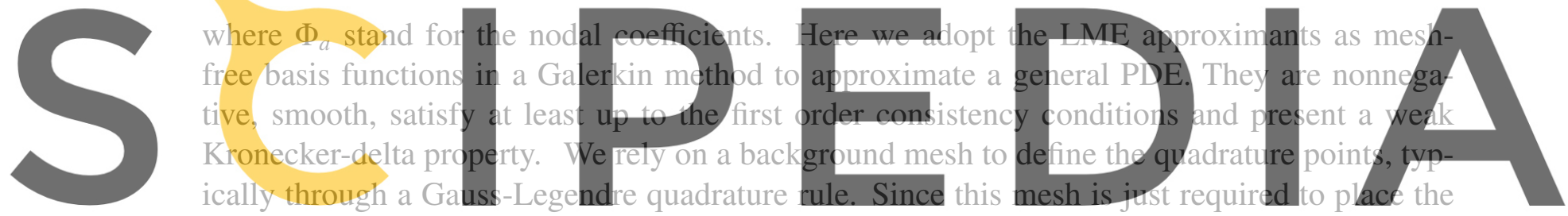

Gauss points, its requirements are less strict than in a mesh-based method. We use here meshes

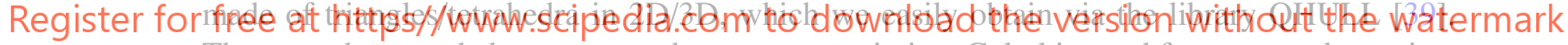

The procedure needed to compute the system matrix in a Galerkin meshfree approach requires mainly four steps: (i) neighborhood search, (ii) computation of the basis functions, (iii) creation of the sparse matrix structure and (iv) Gauss point-wise matrix filling. The pseudocode shown in Algorithm 1 summarizes these four steps. In the present work, we do not deal with solver performance. In the following we briefly extend on the computational implications of every step.

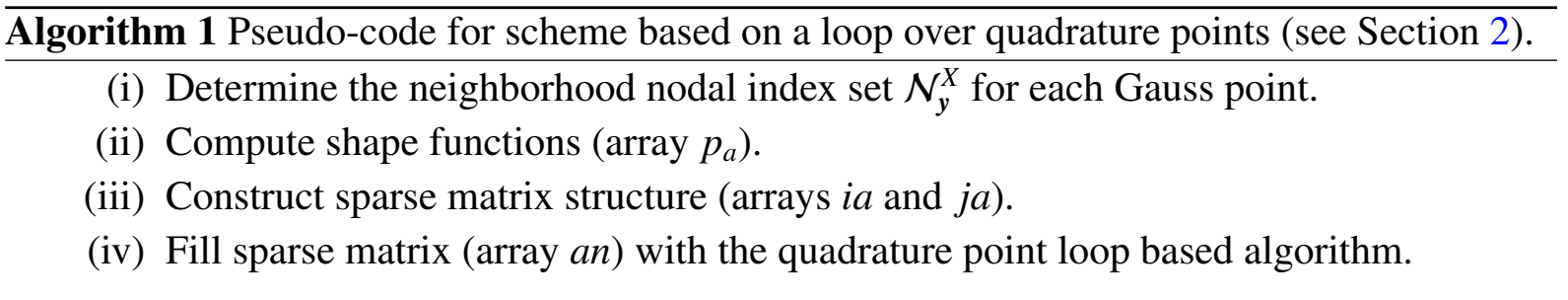

The objective of step (i) is to compute the so-called neighbor lists, which can be interpreted as the counterpart of the mesh connectivity in FEM where the neighbor lists are given by the 
mesh itself. In a meshfree scheme this is made by specialized algorithms, i.e. neighbor searchers which identify the relationship between the quadrature points and the nodes. We will refer to the neighbor lists as primal and dual lists [28], which are complementary. In particular, a dual list identifies the quadrature points that are influenced by a particular node i.e. the quadrature points falling within the effective support of a nodal basis function. Conversely, the primal list contains the nodes that influence a particular quadrature point.

Formally, let $Y=\left\{\boldsymbol{y}_{1}, \boldsymbol{y}_{2}, \ldots, \boldsymbol{y}_{L}\right\} \subset \Omega$ be a set of quadrature points. The dual list containing the nearest points from $Y$ associated with a node $\boldsymbol{x}_{a} \in X$ can be defined as follows

$$
\widetilde{\mathcal{N}}_{\boldsymbol{x}_{a}}^{Y}=\left\{k \in\{1,2, . ., L\}|| \boldsymbol{y}_{k}-\boldsymbol{x}_{a} \mid<r_{a}\right\},
$$

where $r_{a}$ is the effective support radius of the shape function associated to the $a$-th node (which is determined by a user defined cutoff value Tol ${ }_{0}$, see Fig. 1). In the same way, the primal list containing the nodes from $X$ associated with a particular quadrature point $\boldsymbol{y}_{k} \in Y$ is defined as

$$
\mathcal{N}_{y_{k}}^{X}=\left\{a \in\{1,2, . ., N\}|| y_{k}-x_{a} \mid<r_{a}\right\}
$$

The primal and dual lists are used afterwards in steps (ii), (iii) and (iv) to compute the basis functions, identify the nonzero positions in the sparse matrix and perform the assembly. Both lists can be obtained by simply invoking a neighbor scarcher. The neighbor finding problem
is standard, and comes in two flavors, namely finding the $k$-first neighbors or finding neigh-
bors within a range. In our codes, we resort to the approximate neargst neighbor searghing
library [40], whose computational cost scales as $O(N \log N)$, where $N$ is the number of nodes.
In step (iii) the nonzero elements in the global matrix are identiffed using algorithms that postprocess the neighbor lists. This information is critical to properly store the matrix in a sparse

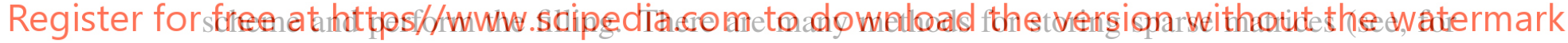
instance, [41] and [42]). We follow here the compressed sparse row (CSR) storage, which is a proper choice for codes written in $\mathrm{C} / \mathrm{C}++$ due to its memory layout. In CSR, a matrix is given in terms of three lists. The first list, $i a$, is an array of integers that stores the total number of nonzeros up to each row. Its dimension is the number of rows plus one, the first position being filled with a zero. The second and third lists are arrays of integers and doubles, ja and an, have as dimension the number of nonzeros in the matrix, and store the column index position and the associated matrix entry. We understand the sparse matrix structure creation as the collection of algorithms required to obtain the lists $i a$ and $j a$. An efficient way to compute this structure based on a loop over quadrature points is presented in Appendix C. The standard algorithm loops over the primal lists of the Gauss points associated to each nodal dual list, such that the nonzero entries of the sparse matrix are identified when two nodes appear together in at least one primal list. As a result, the sparse structure construction becomes increasingly expensive as the number of quadrature points and the support of the basis functions becomes larger.

In step (iv) the nonzero positions of the system sparse matrix (an) are filled in an operation dependent on the PDE. Pursuing a rational memory access, standard Galerkin meshfree algorithms rely on a loop over the Gauss points, each contributing with a local dense matrix. The 
number of rows of this local matrix is equal to the cardinality of the primal list $\left|\mathcal{N}_{y}^{X}\right|=n$ times the number of scalar fields in the problem. Again, the computing time of this step is directly penalized by the increase of quadrature points and by the support size of the basis functions. Furthermore, the local matrices have to be assembled into the global matrix. Since the global matrix is sparse, a search is required to identify the global position to be filled. This concept is illustrated in the upper part of Fig. 3.

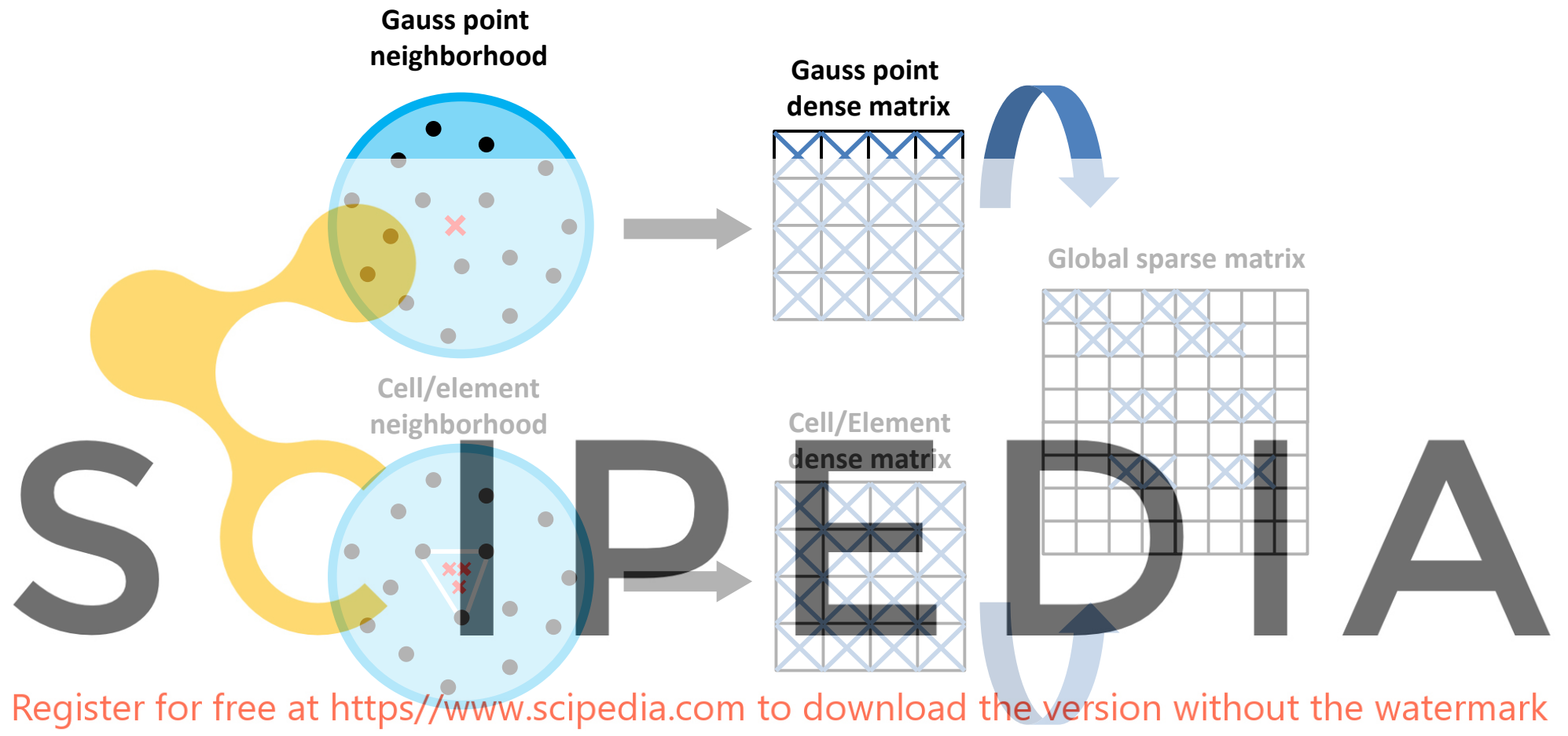

Figure 3: Filling algorithm from neighbor lists to global sparse matrix. Nodal list of neighbors (black dots) can be computed for an individual integration point (red X, top) or for a cell/element (white triangle, bottom). Dense submatrices are generated from these neighborhoods and assembled into the global matrix. Cell/element algorithm improves memory management since the resulting dense submatrix condenses information coming from several integration points.

\section{Meshfree optimization concepts}

We present here two optimizations to improve the efficiency when facing the bottlenecks described in Section 1. First we describe in Section 3.1 a neighborhood coarsening algorithm that considerably speed-up steps (iii) and (iv). Finally, a reduced storage strategy that mitigates the memory requirements when storing the basis functions is detailed in Section 3.2.

\subsection{Neighborhood coarsening algorithm}

A simple idea to alleviate the computational cost of the global sparse matrix is to coarsegrain the neighbor primal lists. The key point is to generate a list for each cell/element of a 
defined coarsening mesh rather than one per Gauss point. The coarsening mesh provides us with a structure to group the primal lists of the Gauss points contained in the cell/element. Without loss of generality, a straightforward and natural choice for the coarsening mesh is the quadrature mesh cells/elements needed in most Galerkin meshfree methods to perform the numerical integration. In this way the complexity added by the increase of Gauss points due to accuracy requirements is removed and the neighbor lists are generated disregarding the number of integration points. We present next details of this procedure.

Once the coarsening mesh is set, we start with a neighbor search over the nodes defining the mesh. This allows us to obtain nodal-based primal lists rather than primal lists for quadrature points. To obtain the cell/element primal lists, the primal lists of its associated nodes are simply merged. More specifically, we define

$$
\mathcal{N}_{e l}=\bigcup_{a \in \mathcal{T}_{e l}} \mathcal{N}_{x_{a}}^{X}
$$

where $\mathcal{T}_{e l}$ is an index set containing the nodal indexes of the $e l$-th cell/element (e.g the mesh connectivity). Note that the $\mathcal{N}_{e l}$ list is applicable to all of the integration points inside the cell/element, regardless their number. This merging operation is negligible in terms of com-
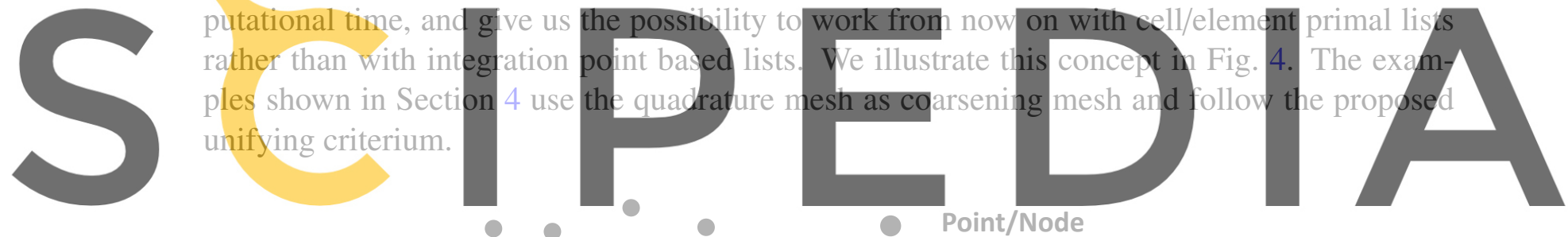

Register for free at https//www.scipedia.com to downloă Gausp point versjon without the watermark

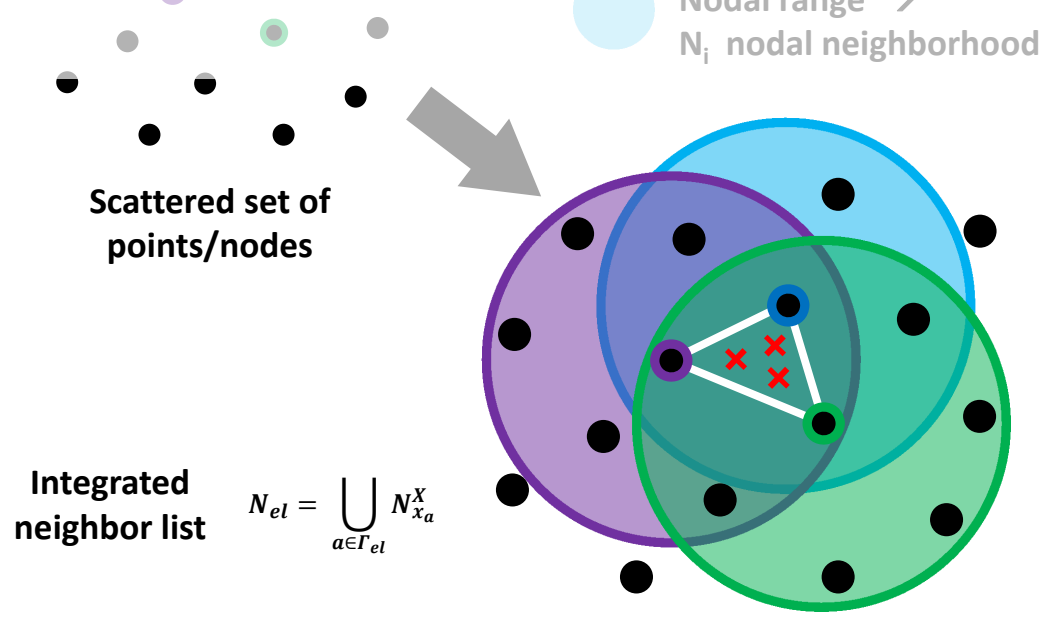

Figure 4: Integrated neighborhood concept. The new cell/element neighborhood is described by the union of nodal vertices lists of neighbors. The triangular elements given by the quadrature mesh are used here as background cell/element generator. 
Vertex merging is a proper strategy when the support size $r_{a}$ is large relative to the mesh size $h_{a}$, which is the usually the case in meshfree methods. Conceivably, it could be the case that the merging of the vertices lists would lead to some loss of information. A node could be influencing an integration point inside a cell/element without influencing any of the vertices containing it, e.g. in highly distorted triangles in 2D. If these unlikely events need to be absolutely ruled out, it is always possible to construct the unified lists by merging the neighbor lists of the Gauss points belonging to a cell/element. In our experience, however, this never happens when using a quadrature mesh; the agreement in numerical integration benchmarks is perfect, and for this reason we recommend the proposed vertex merging to create the cell/element lists.

The creation and filling algorithms can be now based on cell/element neighbor lists, which greatly speeds-up the computations. The structure creation is simplified since only the nodes and cells/elements are involved in the whole procedure. Now the nonzero positions are identified by looping over cell/element neighbor lists instead of looping over Gauss points neighbor lists. The filling of the matrix benefits in two distinct ways. Firstly, the element-wise approach leads to cell/element dense matrices. These local matrices are efficiently filled since just a loop over the neighbor list of the cell/element and a loop over the cell/element Gauss points are required. Secondly, only one dense cell/element matrix is assembled into the global matrix, hence the memory access is improved, as illustrated in the lower part of Fig.

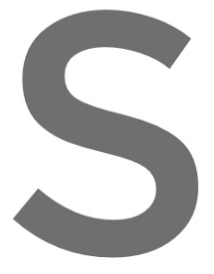

As we show later time associated with points used. This fact ods, namely the large approximants. Furthermore,
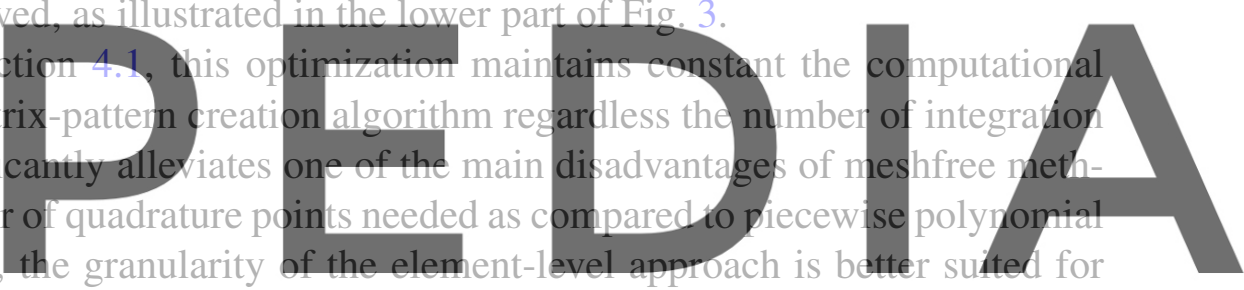

parallel computing, minimizing memory access and limiting data exchange. The pseudo-code

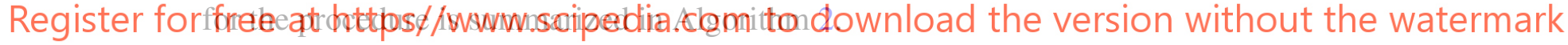

Algorithm 2 Pseudo-code for scheme based on a loop over cells/elements (see Section 3).

(i) Compute adjacency lists for nodes $\mathcal{N}_{x_{a}}^{X}$ and process cell/element lists $\mathcal{N}_{e l}=$ $\bigcup_{a \in \mathcal{T}_{e l}} \mathcal{N}_{x_{a}}^{X}$.

(ii) Compute shape functions (array $p_{a}$ ).

(iii) Construct sparse matrix structure (arrays $i a$ and $j a$ ).

(iv) Fill sparse matrix (array an) with the cell/element loop based algorithm.

\subsection{Compressed meshfree basis functions storage}

A standard practice in the numerical treatment of PDEs is to store in memory the basis functions and their derivatives at each Gauss point. This strategy decreases considerably the computational cost in problems involving nonlinear iterative solvers or evolution problems on Lagrangian meshes. While this storage is insignificant in FEM, in meshfree methods the amount of memory (as quantified later) and its access can become a bottleneck and substantially reduce 
the code efficiency. Here we propose a storage concept that is based on finding structures that optimally synthesize the basis function information at each integration point, striking a tradeoff between memory storage and computation time. We generate data structures that store only partial information about the basis functions and an algorithm to reconstruct them when needed, reducing considerably the memory usage at the expense of a marginal increment in the overall computational cost.

For a general meshfree method, and considering the Galerkin approximation of a fourthorder PDE, the full storage of the basis functions requires $M_{F S}=L \cdot \bar{n} \cdot[1+d+d(d+1) / 2]=$ $L \cdot \bar{n} \cdot\left(1+\frac{3}{2} d+\frac{1}{2} d^{2}\right)$ doubles. In this equation, $L$ is the total number of quadrature points, $\bar{n}$ is the mean cardinality of the primal lists, 1 accounts for the basis functions themselves, $d$ for their gradients, and $d(d+1) / 2$ for the Hessian, which is a symmetric matrix. In a for fourth-order PDE we typically have $\bar{n} \approx 65$ in $2 \mathrm{D}$ and $\bar{n} \approx 380$ in $3 \mathrm{D}$. As a result, the memory requirements rapidly become unaffordable.

Focusing on LME approximants, we recall that the basis functions are obtained by means of a nonlinear optimization problem at each evaluation point with $d$ unknowns, where $d$ is the spatial dimension. This optimization problem yields the Lagrange multiplier associated with first-order consistency conditions. Once the Lagrange multiplier is known, an explicit expression for the basis functions, its gradient and its Hessian is explicit (see Appendix A). Even if the nonlinear optimization problem accounts for a significin A straight-forwarch reals in the Lagrang of the explicit formu
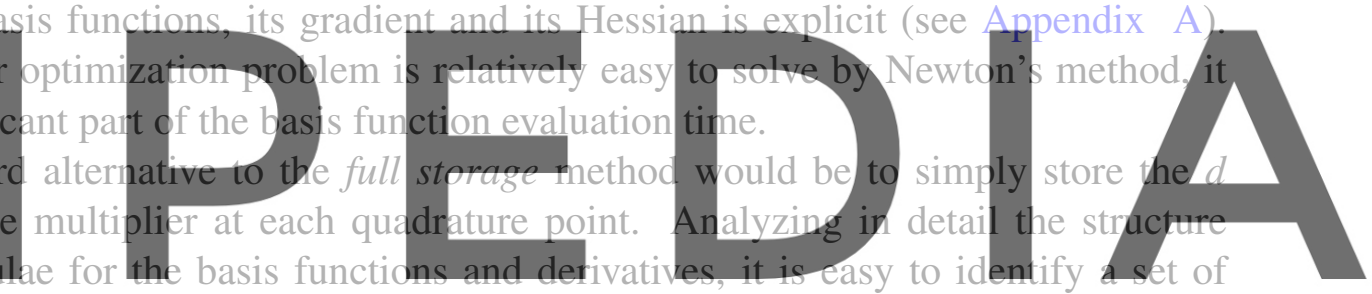

matrices and vectors whose size is independent on $\bar{n}$, and some of which involve summations

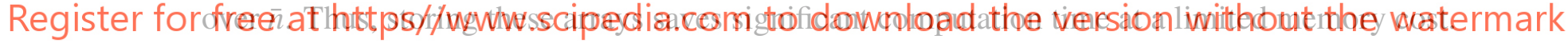

As detailed in Appendix B, this simple observation suggests the optimal or compressed storage, which only involves $M_{O S}=L \cdot\left(2+\frac{7}{2} d+2 d^{2}+\frac{1}{2} d^{3}\right) \approx L \cdot(2+d) \cdot\left(1+\frac{3}{2} d+\frac{1}{2} d^{2}\right)$ doubles. As the mean cardinality is in general much greater than the spatial dimension, i.e. $\bar{n} \gg(2+d)$, from the ratio $M_{F S} / M_{O S}=\bar{n} /(2+d)$ it is clear that the memory usage decreases significantly when the compressed storage technique is used, as can be observed in Table 1.

In Section 4.2 we apply this strategy to a fourth-order PDE requiring the storage of the values, gradients and Hessians of the LME basis functions. We quantify the memory usage and computational time devoted to evaluate the basis functions for both the full and for the optimized storage implementations.

\section{Numerical examples}

The performance of the optimizations presented in Section 3 are studied here in two boundaryvalue problems. We compare the proposed implementation of Galerkin meshfree methods with the standard implementation in terms of storage and computational time. Since we only change the way operations are organized and information stored, the numerical solutions with both 
Table 1: Quantification and comparison of memory usage between the methods of full and optimal or compressed storage of local maximum-entropy basis functions and their derivatives. Here, $\bar{n}$ is the mean cardinality of primal lists, $L$ is the number of Gauss points and $d$ is the spatial dimension.

\begin{tabular}{|c|c|c|}
\hline & Full storage & Optimal storage \\
\hline Total memory usage & $M_{F S}=L \cdot \bar{n} \cdot\left(1+\frac{3}{2} d+\frac{1}{2} d^{2}\right)$ & $M_{O S}=L \cdot\left(2+\frac{7}{2} d+2 d^{2}+\frac{1}{2} d^{3}\right)$ \\
& $\bar{n} \gg(2+d)$ & $M_{O S} \approx L \cdot(2+d) \cdot\left(1+\frac{3}{2} d+\frac{1}{2} d^{2}\right)$ \\
\hline Comparison & \multicolumn{2}{|c|}{$M_{F S} / M_{O S} \approx \bar{n} /(2+d) \gg 1$} \\
\hline
\end{tabular}

implementations are indistinguishable. We focus in the four steps presented in Section 2 and leave aside the solver stage. As we specify in Section 1, in our experience the analized steps can be comparable in computational time to the solver in $2 \mathrm{D}$ problems and exceed it in 3D. In the first example the neighbor coarsening procedure from Section 3.1 is applied to a 2D heat diffusion and a 3D linear elasticity problems, whereas the compressed basis functions storage detailed in Section 3.2 is exercised in a nonlinear fourth-order phase-field PDE. Both problems use uniform grids that ensure a quite constant number of nodal neighbors for every integration

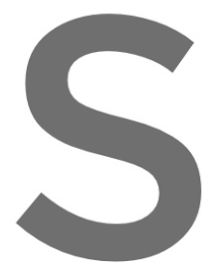
point and facilitate th vertex merging approa stages can be as expr three-dimensional

4.1. The neighborhood coarsening algorithm in 2D and $3 D$ problems

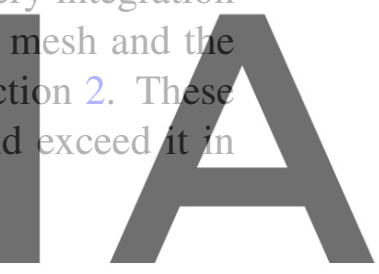

Register for fre

$2 \mathrm{D}$, which is a scalar problem. The sparse matrix structure creation and assembly using the proposed neighborhood coarsening for scalar and vectorial problems is detailed in Appendix $\mathrm{C}$, along with a description of the data structures and a $\mathrm{C} / \mathrm{C}++$ pseudo-code.

The diffusion boundary problem is defined as follows:

$$
\begin{aligned}
\Delta T=f, & \text { in } \Omega, \\
T=T_{0}, & \text { on } \Gamma_{D},
\end{aligned}
$$

where $T$ is the temperature field, $\Omega=(0,1) \times(0,1)$ the domain, $f$ is an arbitrary source of heat and $T_{0}$ is the prescribed temperature on the Dirichlet's boundary $\Gamma_{D}$. We consider $T_{0}=0$ on $\Gamma_{D}=\partial \Omega$ and assume a source $f=2 y$. The solution obtained using LME approximants with a uniform grid of points of $100 \times 100$ is depicted in Fig. 5 .

The entries of the stiffness matrix take the standard form

$$
K_{a b}=\int_{\Omega} \nabla p_{a} \cdot \nabla p_{b} d \Omega
$$



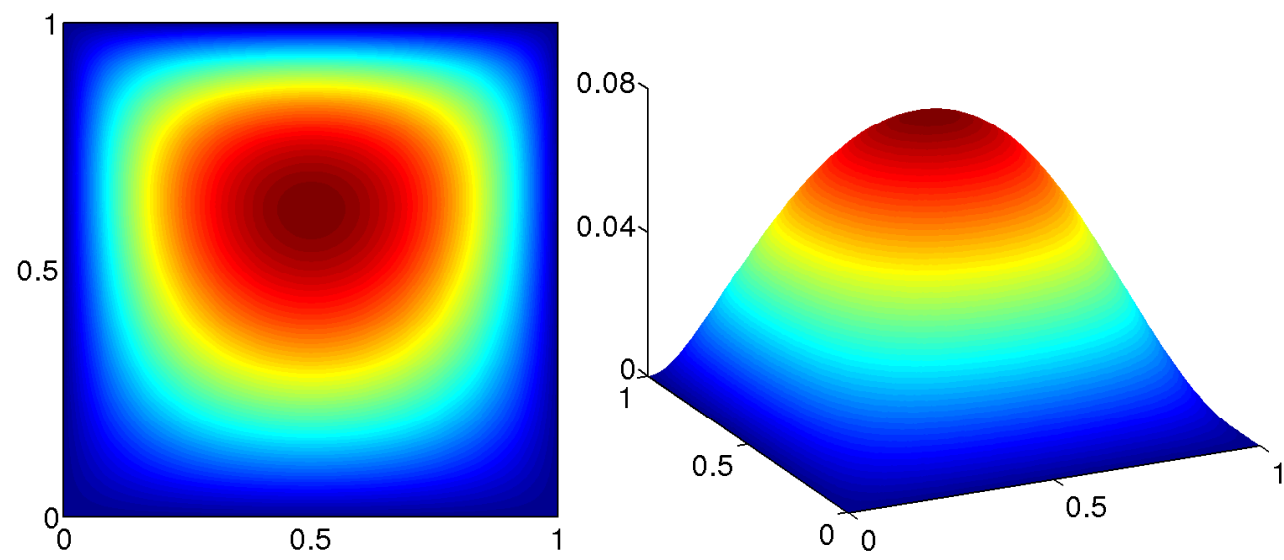

Figure 5: 2D and 3D views of the solution for a heat equation with a source. We use LME approximants, a uniform mesh of $100 \times 100$ nodes, $\gamma=1.6$ and 6 Gauss points per triangular cell/element.

where $\Omega$ cannot be reduced to a set of elements as in FEM. A quadrature rule is defined on a background integration mesh over the whole domain (that may or may not coincide with the coarsening mesh) as

$$
K_{a b}=\sum_{k=1}^{L} \nabla p_{a}\left(\boldsymbol{y}_{k}\right) \cdot \nabla p_{b}\left(\boldsymbol{y}_{k}\right) \omega_{k},
$$

where $\omega_{k}$ stand for the Gauss points quadrature weights in physical space.

The performance of the algorithm based on looping over quadrature points depends on the number of nodes used to discretize the domain, the number of Gauss points per quadrature cell and the size of the support of the basis functions (linked to the aspect ratio parameter $\gamma$ ). In Fig. 6 we show a representative performance, reporting the computational time spent in the main four stages of the algorithm described in Section 2, i.e. (i) neighborhood search, (ii) shape functions, (iii) matrix structure creation and (iv) matrix structure filling. The plots show computational time vs degrees of freedom (number of nodes) for different combinations of the aspect ratio parameter $\gamma=0.8,1.6,4.0$ and three and twelve Gauss points per element. The left-upper chart shows FEM-like LME approximants $(\gamma=4.0)$ with three points per integration element. In this case, the bottleneck in the shape functions calculation. In the other plots we can see how increasing the support size (decreasing $\gamma$ ) or/and increasing Gauss points per element dramatically rises the cost of structure creation and structure filling in comparison with the FEM-like shape functions. The upper charts in Fig. 7 illustrate the computing time growth for different number of Gauss points, whereas the lower charts depict the growth when changing the parameter $\gamma$. The results of both figures highlight the need for speed-up techniques in steps (iii) and (iv) when the size of the system increase for spread-out basis functions $(\gamma=0.8$, large support) that require accurate numerical integration.

We proceed then to analyze the proposed cell/element scheme and review the performance of critical stages (iii) and (iv). In the upper part of Fig. 8 the computational time vs number of 

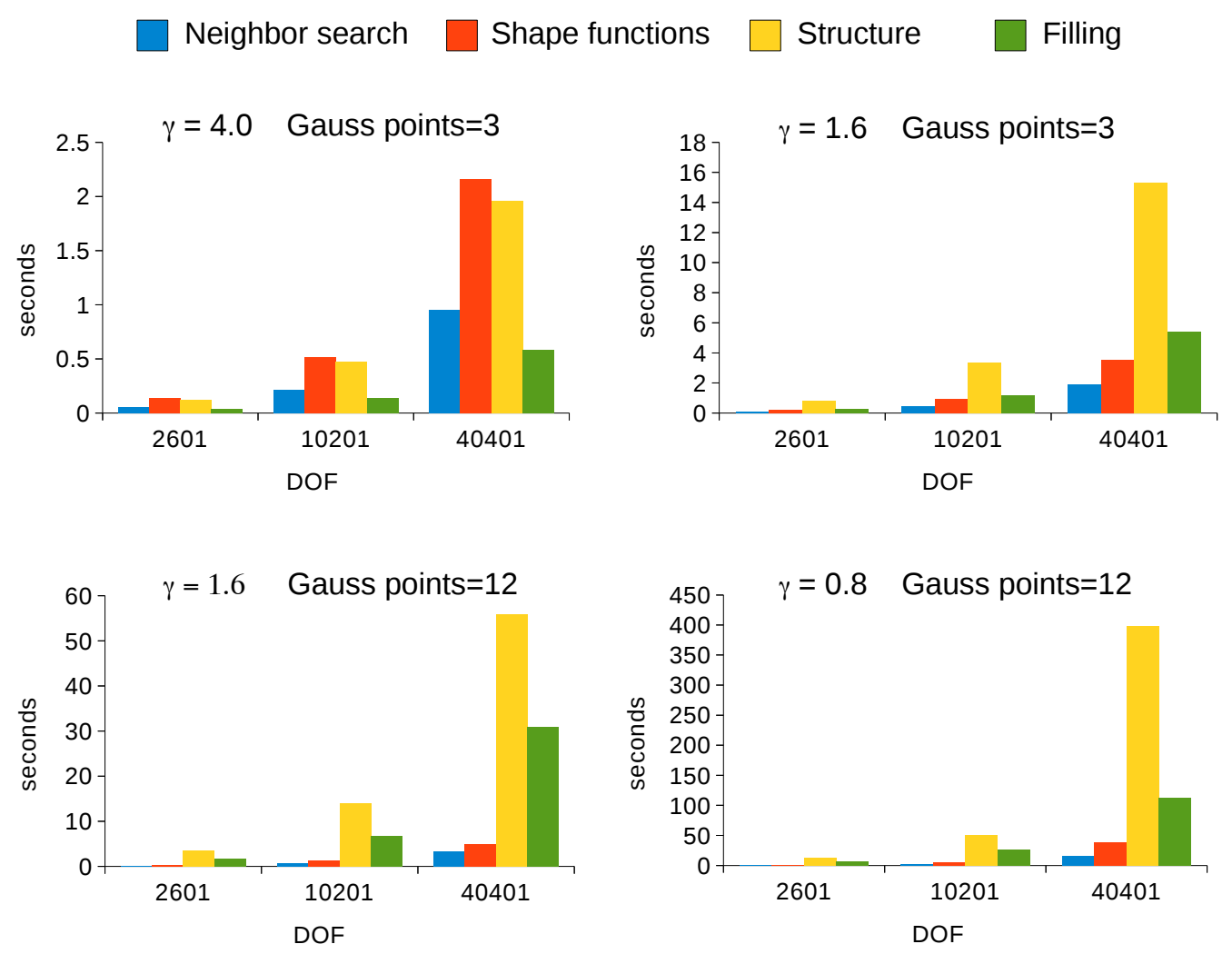

Figure 6: Computational time vs grid size for different values of Gauss points per cell and $\gamma$. From left to right, bars correspond to stages: (i) neighborhood search, (ii) shape functions, (iii) matrix structure creation and (iv) matrix structure filling.

Gauss points is shown for $\gamma=0.8,1.6,4.0$. The juxtaposed bars in the figures compare the standard and the new implementations. We can see how the gain in performance grows as the support size increases, giving greater speed-ups as $\gamma$ decreases e.g. ten times faster for twelve Gauss points and $\gamma=0.8$. Notice also that the matrix structure creation is completely insensitive in the new implementation to the number of quadrature points per element, see Fig. 8. No speed-up is observed when a small number of integration points is used. We show in the lower panels of Fig. 8 the filling algorithm computational time vs number of Gauss points for $\gamma=0.8,1.6,4.0$. We observe the same pattern of speed-ups when $\gamma$ decreases. Notice that although the speed-up is considerable, the filling operations do depend on the number of quadrature points in the nee algorithm, but far less critically than in the standard implementation. Nevertheless, the filling time is greatly reduced with the proposed approach, particularly for large supports i.e. five times smaller for $\gamma=0.8$ and twelve Gauss points.

Finally, the growth of computational time as a function of system size is presented in Fig. 9. The standard implementation using twelve Gauss points is shown as reference. We conclude that our proposal is significantly more efficient than the algorithm based on looping over quadrature points, and that the improvement increases as the number of quadrature points and support 

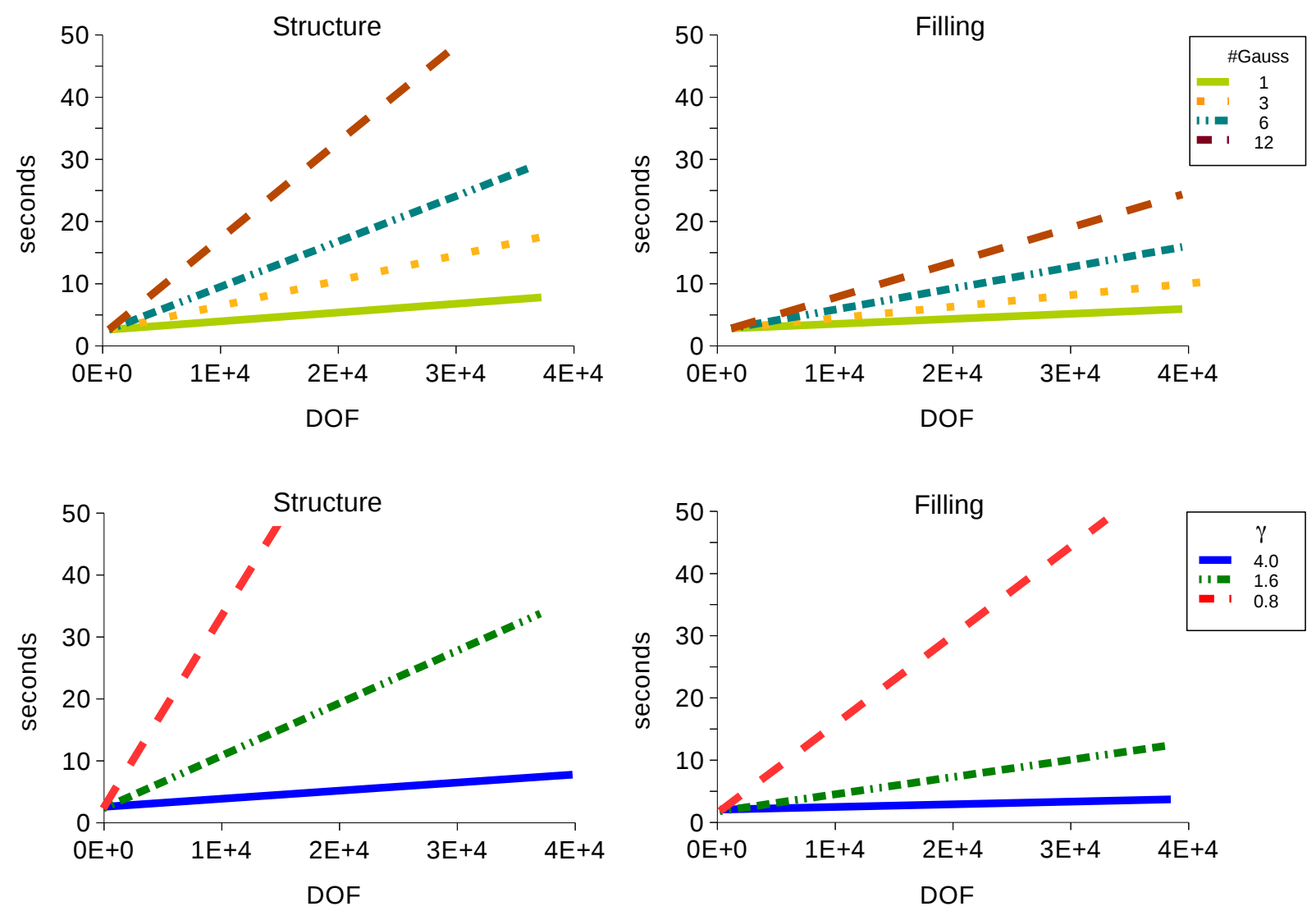

Figure 7: Comparison between growth for matrix structure creation (left) and filling algorithms (right) with the grid size for different values of Gauss points per cell (top, $\gamma=1.6$ ) and for different values of parameter $\gamma$ (bottom, Gauss points $=6$ ).

size become larger.

We test now the neighborhood coarsening algorithm in a 3D linear elasticity problem, where a cube of $l=1.0 \mathrm{~m}$ is stretched (we consider a Young's modulus of $E=0.01 \mathrm{GPa}$ and Poisson's ratio $v=0.2$ ). We present the results for a $20 \times 20 \times 20=8000$ node set with 4,11 and 15 Gauss points per quadrature cell. We summarize in Fig. 10 the results. The time invested in the creation of the structure, which is omitted in the figure, is small $(4 \mathrm{~s})$ and remains constant regardless of the Gauss point number. We focus here in the filling time, which is dominant in $3 \mathrm{D}$, and show that the proposed method is quite insensitive to the number of quadrature points, while the computational time for standard implementation rapidly increases with the number of quadrature points. The filling time for 15 quadrature points is 4 times longer with the standard implementation. Solver time for this problem is around $100 \mathrm{~s}$. 


\section{Standard implementation (structure) $\square$ Cell/element implementation (structure)}
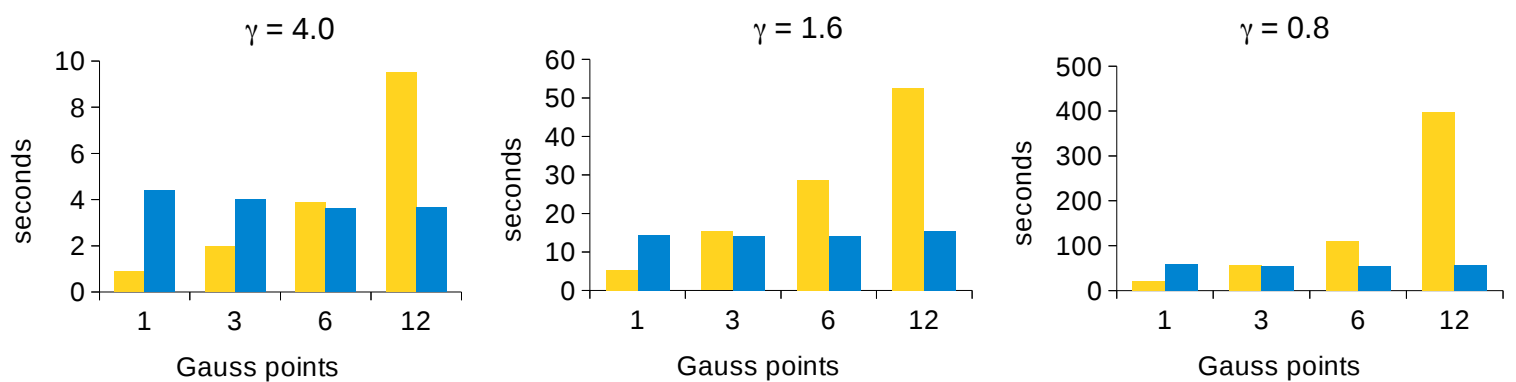

$\square$ Standard implementation (filling) $\square$ Cell/element implementation (filling)
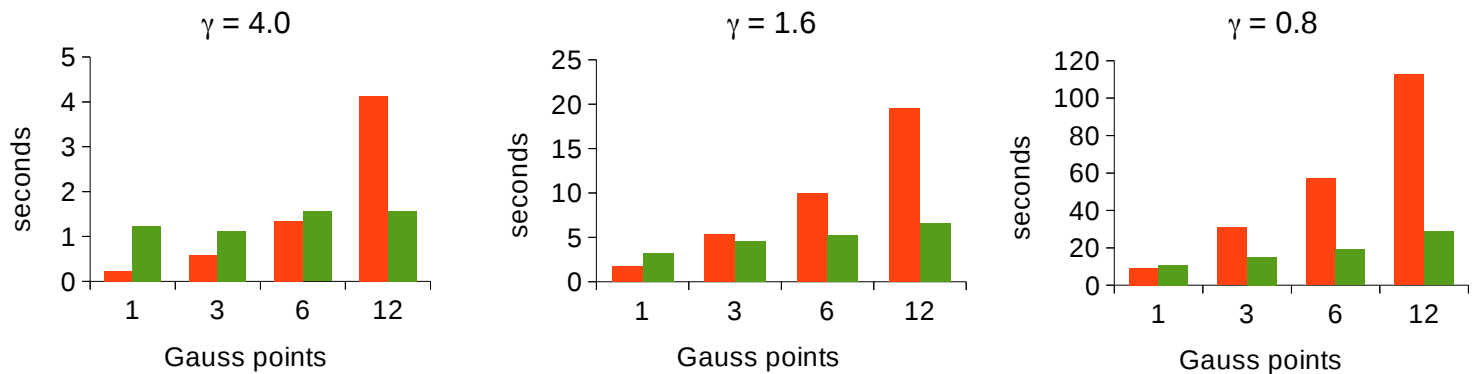

Figure 8: Structure creation (top) and filling (bottom) computational time vs Gauss points for decreasing $\gamma$ (increasing support). Standard and new implementation are shown (left and right bars, respectively). DOF $=40,401$.
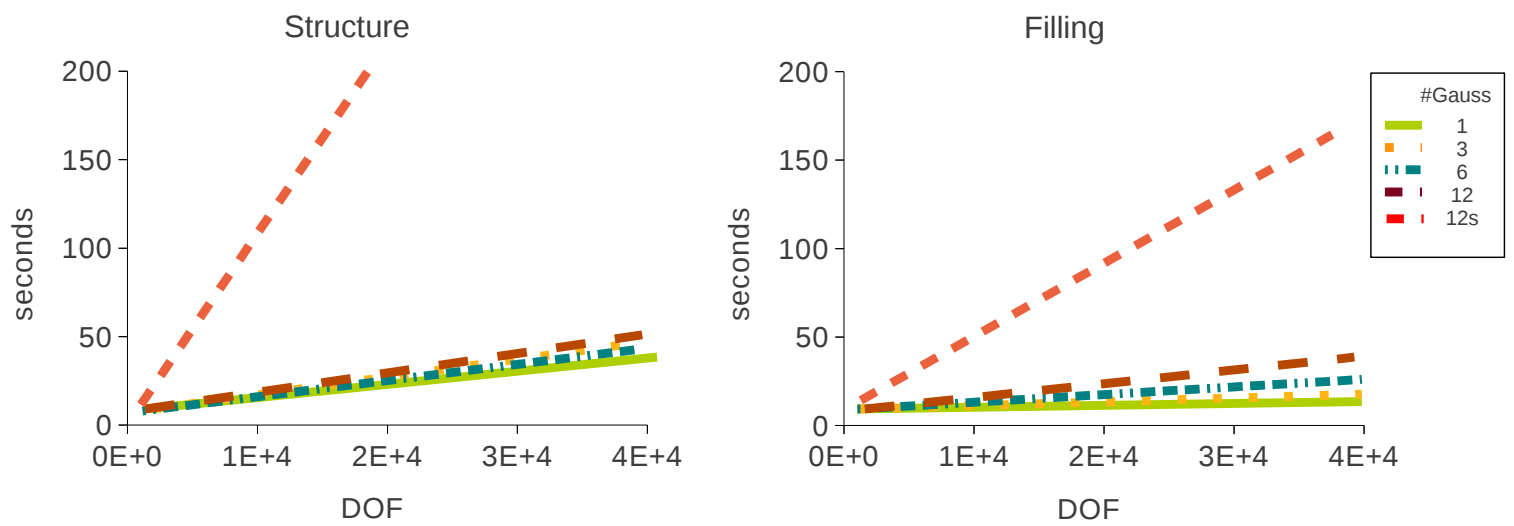

Figure 9: Growth of the computational time as a function of the size of the system for the matrix structure creation (left) and filling (right), using the new implementation different numbers of Gauss points per cell and $\gamma=0.8$. For comparison purposes, the standard implementation using 12 Gauss points (legend 12s) is presented.

\subsection{The compressed meshfree basis functions storage applied to a phase-field fracture model}

We present here the results of the proposed memory storage strategy for the LME approximants. We compare the full storage and optimized schemes in a fourth-order PDE problem 


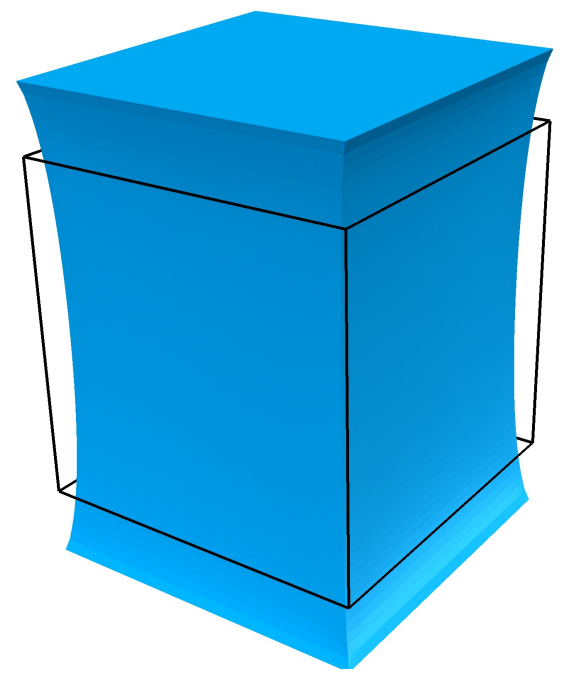

Cell/element implementation (filling)

Standard implementation (filling)

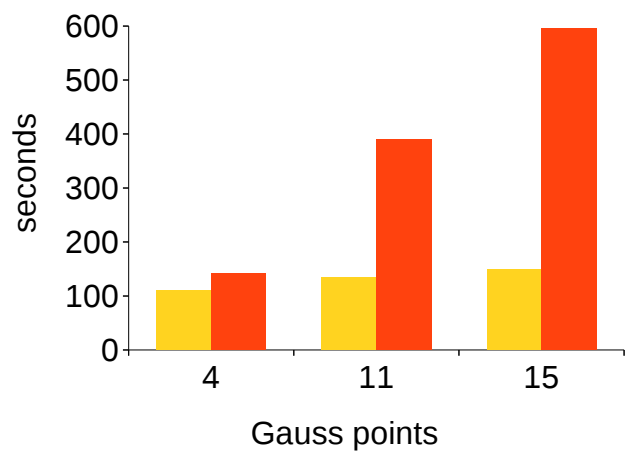

Figure 10: Deformed configuration for 3D linear elasticity benchmark (left). Computational time for the filling algorithm using the new and the standard implementation for different numbers of Gauss points per cell, $\gamma=0.8$ and 24, 000 degrees of freedom.

requiring the values, gradients and Hessians of the basis functions. In a variational model of fracture, the phase-field PDE results from the following functional

$$
\int_{\Gamma} G_{c} d \Gamma=\int_{\Omega} G_{c}\left[\frac{(1-\phi)^{2}}{4 l_{0}}+\frac{l_{0}}{2}|\nabla \phi|^{2}+\frac{l_{0}^{3}}{4} \Delta \phi^{2}\right] d \Omega,
$$

where $G_{c}$ is the critical fracture energy density, $\phi$ the phase-field, and $l_{0}$ the parameter controlling the width of the approximation of the crack. We illustrate a typical solution in Fig. 11. More details about this particular model can be found in [43].

We focus on the amount of doubles that need to be stored when using a standard and the optimized scheme, and also on the impact on computational time of the structure filling routine for the global matrix. The latter requires retrieving the stored basis functions in the usual approach, and partially recomputing them in the optimized storage approach. The structure creation step is completely independent on evaluation/retrieval of the basis functions, and for this reason we do not report it here. As can be observed in the left panel of Fig. 12, the optimized storage strategy decreases the memory requirements by an order of magnitude; the ratio of memory requirements is about 20. For this two-dimensional problem we use $\gamma=1$, leading to a mean value of 72 neighbors per integration point. Here we use six Gauss points by element.

We analyze now the computational time invested in the filling of the global matrix. We can observe in the right panel of Fig. 12 that the memory optimized storage is marginally slower than the standard routine. The extra operations to retrieve the basis functions and its derivatives, see Appendix A, is partially compensated by a more efficient access to the memory, resulting in running time increments of about $10 \%$. 

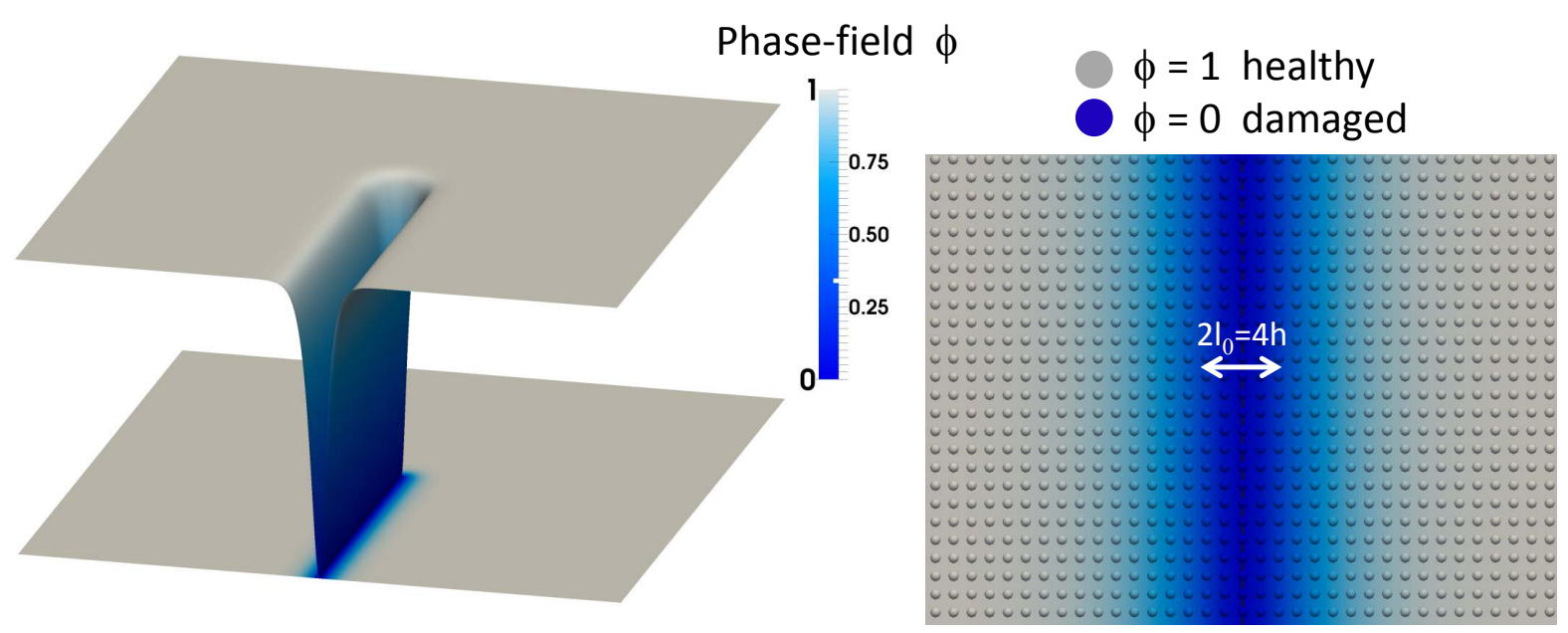

Figure 11: Fourth-order phase-field solution for a crack. Phase-field values range from 1 to 0 signaling the progressive damaging of the material (left). The ratio between the crack width parameter $l_{0}$ and the nodal spacing $h$ is 2 (right).
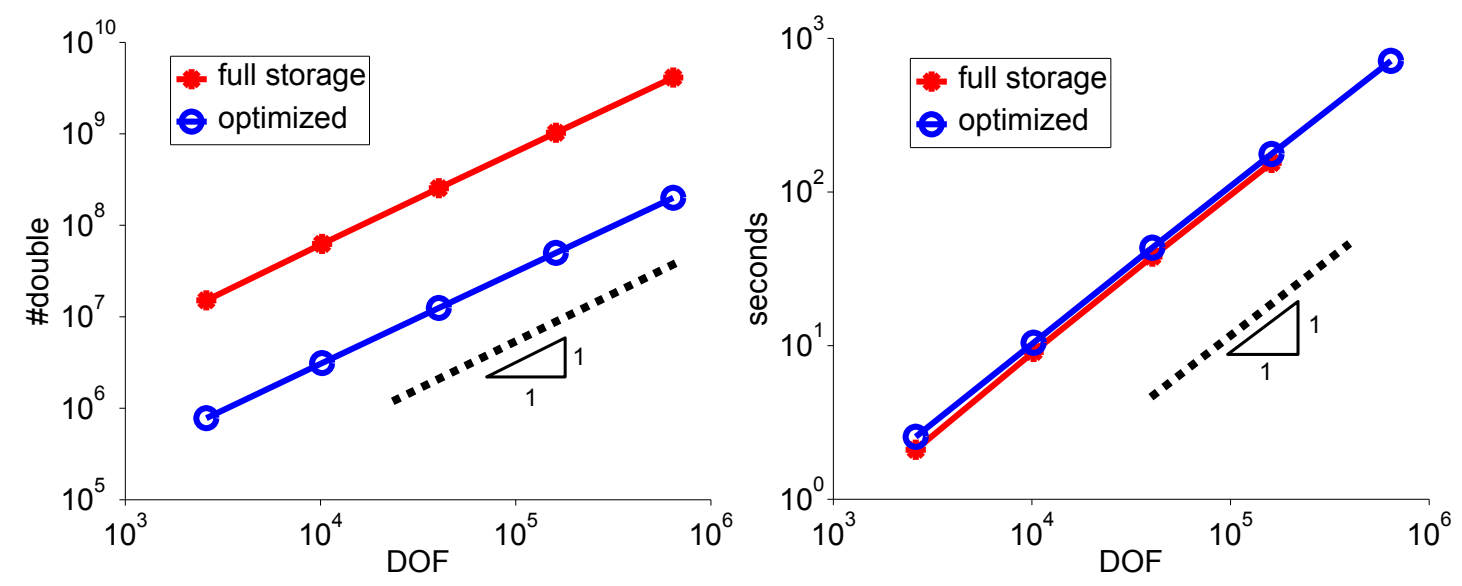

Figure 12: Comparison between the full storage and optimized implementations. The plots show the number of doubles stored in the global matrix vs the number of degrees of freedom (left) and the computational time invested in the filling and assembly of the global matrix vs the number of degrees of freedom (right).

\section{Conclusions}

We have presented two optimization procedures to mitigate two fundamental bottlenecks in Galerkin meshfree methods: matrix assembly and basis functions storage. We have shown how the sparse structure creation and filling of the system matrix become critical in a meshfree context when either the support size of the basis functions or the number of integration points increases. We have introduced a simple coarse-graining procedure for matrix structure creation and filling, where we change from an integration point perspective to one based on cells/elements. As a result of this optimization, the dependence of the computational time on 
the number of integration points is completely severed in the sparse structure creation and dramatically decreased in the matrix assembly. We tested the new implementation on a 2D heat diffusion PDE and 3D linear elasticity problem, speeding-up ten times the structure creation and five times the filling in the case of twelve(2D)/fifteen(3D) Gauss points and $\gamma=0.8$. Additionally, a compressed memory storage for LME approximants has been introduced to alleviate memory requirements. We have shown how this methodology can recover with minimal computational overhead the basis functions, gradients and Hessians that are repeatedly required in large-scale nonlinear or evolution problems, hence reducing drastically the amount of memory by 20-fold for a scalar fourth-order PDE in 2D.

Further research in Galerkin meshfree methods should focus on tridimensional problems and the study of proper parallelization algorithms for supercomputing. We have successfully parallelized the presented techniques using state-of-the-art scientific codes such as PETSc (portable, extensible toolkit for scientific computation library, [44]) and ParMetis [45] for reordering and partitioning. Our current experience on a supercomputing facility further highlights the importance of optimizations such as those presented here in large-scale vectorial problems in 3D. Models showing an intrinsic high computational cost such as the phase-field approaches can particularly benefit from this concept due to the easy parallelization of the algorithms presented. The approximation of phase-field models with LME in biomembrane dynamics $[32,33]$ and fracture mechanics $[35,36]$ are successful examples of these optimization procedures.

\section{Acknowledgments}

We acknowledge the support of the European Research Council under the European Community's 7th Framework Programme (FP7/2007-2013)/ERC grant agreement nr 240487, and of the Ministerio de Ciencia e Innovación (DPI2011-26589). CP acknowledges FPI-UPC Grant and FPU Ph. D. Grant (Ministry of Science and Innovation, Spain).

\section{Appendix A. Optimal storage of local maximum-entropy approximants}

We review here the calculation of local maximum-entropy (LME) basis functions and their derivatives. We represent spatial gradients of scalar functions by $\nabla$, and we denote by $\boldsymbol{D} \boldsymbol{f}(\boldsymbol{x})$ the matrix of partial derivatives for vector-valued functions. The subindexes $a$ and $b$ refer to nodes. Summation is not implied for repeated node indices (see $[22,25,28]$ for further explanation).

Let $X$ be a set of $N$ scattered nodes $X=\left\{\boldsymbol{x}_{1}, \boldsymbol{x}_{2}, \ldots, \boldsymbol{x}_{N}\right\} \subset \mathbb{R}^{d}$, where $d=1,2,3$ is the spatial dimension, and their associate set of locality parameters $\left\{\beta_{1}, \beta_{2}, \ldots, \beta_{N}\right\} \subset \mathbb{R}$. Given a point $\boldsymbol{x}$, recall that the primal list $\mathcal{N}_{x}^{X}$ contains the indices of the nodes affecting $\boldsymbol{x}$. The evaluation of the basis function corresponding to the nodal point $a$ is computed as

$$
p_{a}(\boldsymbol{x})=\frac{\exp \left[-\beta_{a}\left|\boldsymbol{x}-\boldsymbol{x}_{a}\right|^{2}+\lambda^{*} \cdot\left(\boldsymbol{x}-\boldsymbol{x}_{a}\right)\right]}{Z(\boldsymbol{x})},
$$


where $Z(\boldsymbol{x})$ is a partition function

$$
Z(\boldsymbol{x})=\sum_{a \in \mathcal{N}_{x}^{X}} \exp \left[-\beta_{a}\left|\boldsymbol{x}-\boldsymbol{x}_{a}\right|^{2}+\lambda^{*} \cdot\left(\boldsymbol{x}-\boldsymbol{x}_{a}\right)\right],
$$

and the Lagrange multiplier $\lambda^{*}$ is the minimizer of the cost function $\ln Z(x, \lambda)$ [22], that is

$$
\lambda^{*}(\boldsymbol{x})=\arg \min _{\lambda \in \mathbb{R}^{d}} \ln Z(\boldsymbol{x}, \lambda) .
$$

The first spatial derivatives of the basis functions (gradient) are computed as [22, 25]

$$
\nabla p_{a}(\boldsymbol{x})=p_{a}\left[\boldsymbol{r}_{\beta}-\boldsymbol{M}_{a}\left(\boldsymbol{x}-\boldsymbol{x}_{a}\right)\right] \in \mathbb{R}^{d},
$$

where

$$
\begin{aligned}
& \boldsymbol{r}_{\beta}(\boldsymbol{x})=2 \sum_{b \in \mathcal{N}_{x}^{X}} \beta_{b} p_{b}\left(\boldsymbol{x}-\boldsymbol{x}_{b}\right) \in \mathbb{R}^{d}, \quad \boldsymbol{M}_{a}=2 \beta_{a} \boldsymbol{I}-D \lambda \in \mathbb{R}^{d \times d}, \quad D \lambda(\boldsymbol{x})=\left(\boldsymbol{J}_{\beta}-\boldsymbol{I}\right) \boldsymbol{J}^{-1} \in \mathbb{R}^{d \times d}, \\
& \boldsymbol{J}_{\beta}(\boldsymbol{x})=2 \sum_{b \in \mathcal{N}_{\boldsymbol{x}}^{X}} \beta_{b} p_{b}\left(\boldsymbol{x}-\boldsymbol{x}_{b}\right) \otimes\left(\boldsymbol{x}-\boldsymbol{x}_{b}\right) \in \mathbb{R}^{d \times d}, \text { and } \boldsymbol{J}(\boldsymbol{x})=\sum_{b \in \mathcal{N}_{\boldsymbol{x}}^{X}} p_{b}\left(\boldsymbol{x}-\boldsymbol{x}_{b}\right) \otimes\left(\boldsymbol{x}-\boldsymbol{x}_{b}\right) \in \mathbb{R}^{d \times d} .
\end{aligned}
$$

The second spatial derivatives of the basis functions (Hessian matrix) can be written as [28]

$$
\begin{aligned}
H p_{a}(\boldsymbol{x})= & p_{a}\left[\boldsymbol{r}_{\beta}-\boldsymbol{M}_{a}\left(\boldsymbol{x}-\boldsymbol{x}_{a}\right)\right] \otimes\left[\boldsymbol{r}_{\beta}-\boldsymbol{M}_{a}\left(\boldsymbol{x}-\boldsymbol{x}_{a}\right)\right] \\
& +p_{a}\left[\boldsymbol{r}_{\beta} \otimes \boldsymbol{r}_{\beta}+\boldsymbol{r}_{\beta} \otimes \boldsymbol{j}_{a}+\boldsymbol{j}_{a} \otimes \boldsymbol{r}_{\beta}+\left(\boldsymbol{r}_{\beta} \cdot \boldsymbol{j}_{a}\right) \boldsymbol{I}\right] \\
& +p_{a}\left[2\left(\bar{\beta}-\beta_{a}\right) \boldsymbol{I}-\boldsymbol{Q}-\boldsymbol{j}_{a} \cdot \boldsymbol{T}\right] \quad \in \mathbb{R}^{d \times d}
\end{aligned}
$$

where

$$
\begin{gathered}
\boldsymbol{J}_{a}=\boldsymbol{J}^{-1}\left(\boldsymbol{x}-\boldsymbol{x}_{a}\right) \in \mathbb{R}^{d}, \quad \boldsymbol{Q}(\boldsymbol{x})=\sum_{b \in \mathcal{N}_{\boldsymbol{x}}^{X}} p_{b} \boldsymbol{M}_{b}\left(\boldsymbol{x}-\boldsymbol{x}_{b}\right) \otimes \boldsymbol{M}_{b}\left(\boldsymbol{x}-\boldsymbol{x}_{b}\right) \in \mathbb{R}^{d \times d}, \\
\bar{\beta}(\boldsymbol{x})=\sum_{b \in \mathcal{N}_{x}^{X}} \beta_{b} p_{b} \in \mathbb{R}, \text { and } \boldsymbol{T}(\boldsymbol{x})=\sum_{b \in \mathcal{N}_{x}^{X}} p_{b}\left(\boldsymbol{x}-\boldsymbol{x}_{b}\right) \otimes \boldsymbol{M}_{b}\left(\boldsymbol{x}-\boldsymbol{x}_{b}\right) \otimes \boldsymbol{M}_{b}\left(\boldsymbol{x}-\boldsymbol{x}_{b}\right) \in \mathbb{R}^{d \times d \times d} .
\end{gathered}
$$

\section{Appendix B. Quantification of memory usage}

We quantify here the memory usage for two different strategies to store local maximumentropy basis functions and their derivatives: the full storage and the optimal or compressed storage methods.

The basis functions are usually computed and stored in memory for a given a set of $L$ quadrature points $\left\{\boldsymbol{y}_{1}, \boldsymbol{y}_{2}, \ldots, \boldsymbol{y}_{L}\right\} \subset \mathbb{R}^{d}$ and the associated set of primal lists $\left\{\mathcal{N}_{\boldsymbol{y}_{1}}^{X}, \mathcal{N}_{\boldsymbol{y}_{2}}^{X}, \ldots, \mathcal{N}_{\boldsymbol{y}_{L}}^{X}\right\}$ (see Section 2). By defining as $n_{k}=\left|\mathcal{N}_{y_{k}}^{X}\right|$ the cardinality corresponding to the primal list of the 
Table B.2: Quantification of memory usage for two methods that store local maximum-entropy basis functions and their derivatives. The optimal or compressed storage (OS) technique needs approximately $L \cdot(2+d) \cdot\left(1+\frac{3}{2} d+\frac{1}{2} d^{2}\right)$ doubles, while the full storage (FS) method demands $L \cdot \bar{n} \cdot\left(1+\frac{3}{2} d+\frac{1}{2} d^{2}\right)$ doubles, where $L$ is the number of quadrature points, $d$ the spatial dimension, and $\bar{n} \gg(2+d)$ the mean cardinality of the primal lists. The ratio $M_{F S} / M_{O S}$ shows that memory usage decreases significantly when the compressed storage technique is used.

\begin{tabular}{|c|c|c|c|c|}
\hline & \multicolumn{2}{|r|}{ Full storage } & \multicolumn{2}{|c|}{ Optimal storage } \\
\hline & Variable & Memory usage & Variable & Memory usage \\
\hline Basis functions & $p_{a}$ & $L \cdot \bar{n}$ & $\begin{array}{l}Z(x) \\
\lambda(x)\end{array}$ & $\begin{array}{c}L \\
L \cdot d\end{array}$ \\
\hline First spatial derivatives & $\nabla p_{a}$ & $L \cdot \bar{n} \cdot d$ & $\begin{array}{l}r_{\beta}(x) \\
D \lambda(x)\end{array}$ & $\begin{array}{c}L \cdot d \\
L \cdot d \cdot(d+1) / 2\end{array}$ \\
\hline Second spatial derivatives & $H p_{a}$ & $L \cdot \bar{n} \cdot d \cdot(d+1) / 2$ & $\begin{array}{l}\bar{\beta}(x) \\
J(x) \\
Q(x) \\
T(x)\end{array}$ & $\begin{array}{c}L \\
L \cdot d \cdot(d+1) / 2 \\
L \cdot d \cdot(d+1) / 2 \\
L \cdot d \cdot d \cdot(d+1) / 2\end{array}$ \\
\hline Total memory usage & $M_{F S}=1$ & $\begin{array}{l}\cdot \bar{n} \cdot\left(1+\frac{3}{2} d+\frac{1}{2} d^{2}\right) \\
i \gg(2+d)\end{array}$ & $\begin{array}{c}M_{O S}= \\
M_{O S} \approx L\end{array}$ & $\begin{array}{l}\left(2+\frac{7}{2} d+2 d^{2}+\frac{1}{2} d^{3}\right) \\
2+d) \cdot\left(1+\frac{3}{2} d+\frac{1}{2} d^{2}\right)\end{array}$ \\
\hline Comparison & & $M_{F S} / M_{O S}$ & $\bar{n} /(2+d$ & \\
\hline
\end{tabular}

quadrature point $\boldsymbol{y}_{k}$, we can construct the set of cardinalities $\left\{n_{1}, n_{2}, \ldots, n_{L}\right\} \subset \mathbb{R}$. To simplify the calculations, we define the mean cardinality as $\bar{n}=\left(\sum_{k=1}^{L} n_{k}\right) / L$.

The full storage (FS) method demands a massive usage of memory because basis functions and first and second derivatives associated to all the nodal points, and evaluated at all the quadrature points, need to be stored in memory. The calculation of memory usage is straightforward from the analysis of Eqs. A.1, A.2 and A.3 (see Table B.2 for a summary): $M_{F S}=L \cdot \bar{n} \cdot(1+d+d(d+1) / 2)=L \cdot \bar{n} \cdot\left(1+\frac{3}{2} d+\frac{1}{2} d^{2}\right)$ doubles, where here and elsewhere we exploit the symmetry of matrices (here the Hessian) to reduce storage. On the other hand, the optimal or compressed storage (OS) method only requires the storage of some specific variables associated to the quadrature points. We quantify the memory usage of this method in Table B.2: $M_{O S}=L \cdot\left(2+\frac{7}{2} d+2 d^{2}+\frac{1}{2} d^{3}\right) \approx L \cdot(2+d) \cdot\left(1+\frac{3}{2} d+\frac{1}{2} d^{2}\right)$ doubles. As the mean cardinality is regularly much greater than the spatial dimension, i.e., $\bar{n} \gg(2+d)$, from the ratio $M_{F S} / M_{O S}=\bar{n} /(2+d)$ we can conclude that the memory usage decreases significantly when the compressed storage technique is used. 


\section{Appendix C. Data structure and specialized algorithms}

We detail here the data structures and algorithms proposed to handle more efficiently sparse matrices in the context of meshfree methods. The data structures are specifically designed to store the neighborhood index sets for particular "entities" (elements, nodes, or quadrature points). The algorithms described are responsible for the creation of the sparse matrix structure and the assembly process.

\section{Appendix C.1. Data structure to store neighbor lists}

The data structure to store neighbor lists is inspired in the compressed sparse row storage format (see Section 2) and consists of two arrays, one indicating the number of neighboring points to an entity (pointer_array), and the other containing the index or identification number of each one of these points (index_array). Depending on the kind of assembly process, we need to construct at least two of the following four neighborhood index sets:

- Primal lists: set of neighboring nodes to each quadrature point. These lists, stored in the arrays $i s \_n$ and $j s \_n$, are required for the assembly process based on a loop over the quadrature points (see Section 2).

- Dual lists: set of the neighboring quadrature points to each nodal point (see Section 2 for details). These lists, stored in the arrays $i n_{-} s$ and $j n_{-} s$, are dual to the primal lists.

- Lists of the neighboring nodal points to each cell/element, stored in the arrays ie $\_n$ and $j e . n$. These sets, defined in Section 3.1, are needed for the assembly process based on a loop over the cell/elements.

- Lists of the neighboring cell/elements to each nodal point (arrays in_e and jn_e). These lists are dual to those contained in the set of arrays $i e \_n$ and $j e \_n$.

We use here the primal lists to explain how the information of the neighborhood index sets is stored in the arrays pointer_array and index_array, which in this work are respectively referred as $i s \_n$ and $j s \_n$. Given a set of $L$ quadrature points $\left\{\boldsymbol{y}_{1}, \boldsymbol{y}_{2}, \ldots, \boldsymbol{y}_{L}\right\} \subset \mathbb{R}^{d}$ and the associated set of primal lists $\left\{\mathcal{N}_{y_{1}}^{X}, \mathcal{N}_{y_{2}}^{X}, \ldots, \mathcal{N}_{y_{L}}^{X}\right\}$, where $d$ is the spatial dimension, $\mathcal{N}_{y_{k}}^{X}=\{a \in$ $\left.\{1,2, \ldots, N\} \mid p_{a}\left(\boldsymbol{y}_{k}\right)>\mathrm{Tol}_{0}\right\}$ the primal list for the quadrature point $\boldsymbol{y}_{k}, N$ the total number of nodes, $\mathrm{Tol}_{0}$ a numerical tolerance, and $p_{a}\left(\boldsymbol{y}_{k}\right)$ the evaluation at the point $\boldsymbol{y}_{k}$ of the basis function corresponding to the node $a$, the information stored in the arrays is the following:

- is $\_n$ : the component $p+1$ of this array is defined as $i s \_n(p+1)=\sum_{k=1}^{p}\left|\mathcal{N}_{y_{k}}^{X}\right|$. In other words, the element (or position) $p+1$ of the array contains the summation of the cardinalities of the primal lists associated with the first $p$ quadrature points. Note that the first component is always zero and the length of the array is $\operatorname{dim}\left(i s \_n\right)=L+1$. 
- $j s \_n$ : this array, which stores consecutively in memory all the primal lists, is defined as $j s \_n=\left(\mathcal{N}_{y_{1}}^{X}, \mathcal{N}_{y_{2}}^{X}, \ldots, \mathcal{N}_{y_{L}}^{X}\right)$. The length of the array is $\operatorname{dim}\left(j s_{-} n\right)=\sum_{k=1}^{L}\left|\mathcal{N}_{\boldsymbol{y}_{k}}^{X}\right|$, where the cardinality can be different for each quadrature point. Note that the order of the quadrature points is important and, in general, $\operatorname{dim}\left(j s \_n\right) \ll N \cdot L$.

\section{Appendix C.2. Algorithms for matrix structure creation and assembly process}

The algorithms implemented to create the sparse matrix structure and the assembly process are presented here in a $\mathrm{C} / \mathrm{C}++$ pseudo-code (declarations are left out for the sake of clarity). The three routines detailed in the subsequent sections are:

- CreateElementBasicStructure1D(): this algorithm creates the arrays $i a 1$ and ja1 of the sparse matrix structure for the case in which the physical field is scalar. The neighbor lists $i s \_n$ and $j s \_n$ are used in the method based on a loop over the quadrature points, and the lists $i e \_n, j e \_n$ in the cell/element scheme.

- CreateStructureND(): extension of the previous algorithm to the n-dimensional case, i.e., when the physical field is vectorial. The arrays created are denoted by ia and ja.

- FillStructureND(): algorithm to fill the array an by executing the operations implemented in the pointer function $* p$ function. The arrays $i a$ and $j a$ are needed in the assembly process to loop over the rows and columns of the sparse matrix.

\section{Appendix C.2.1. CreateElementBasicStructure1D()}

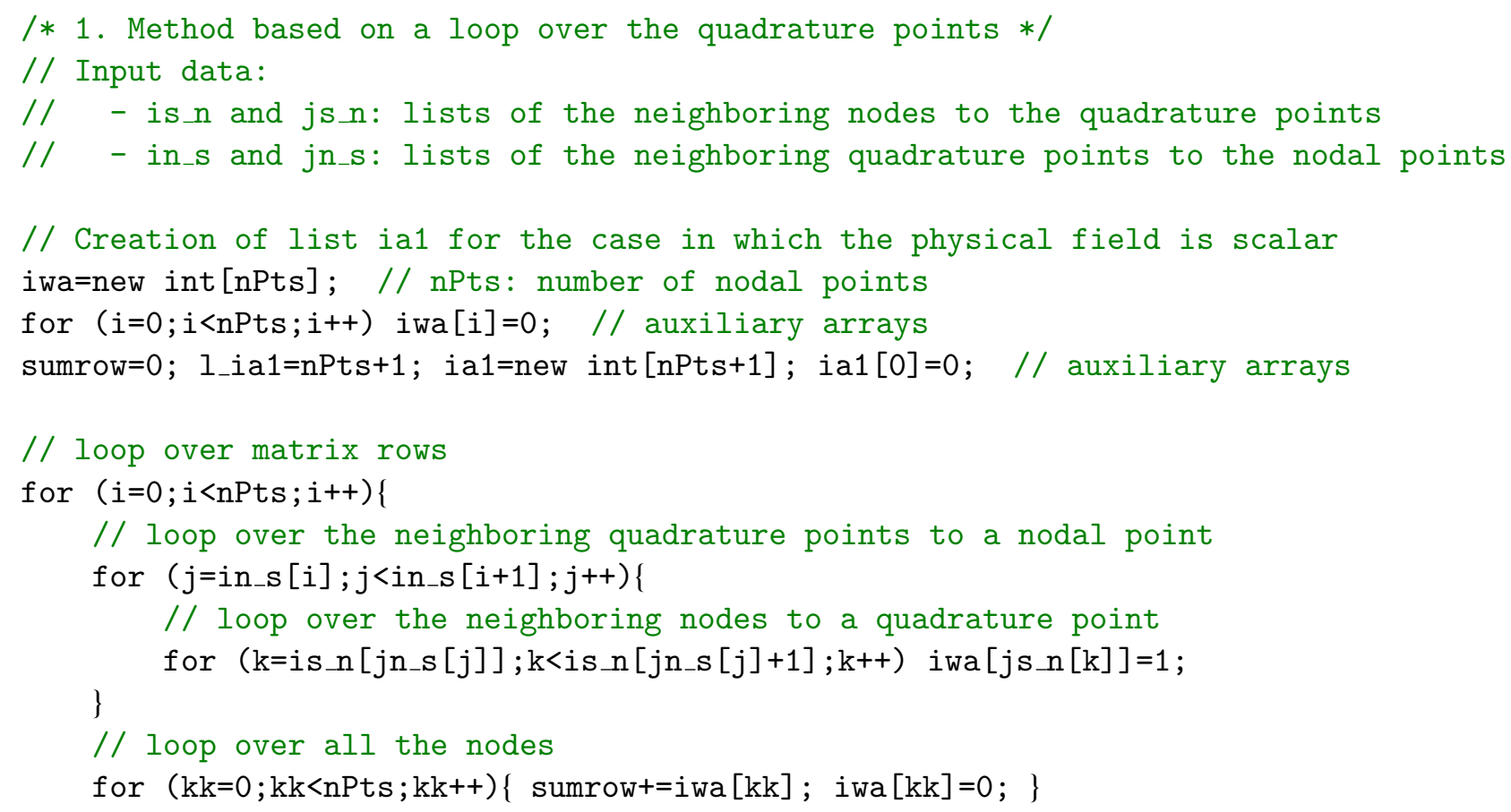




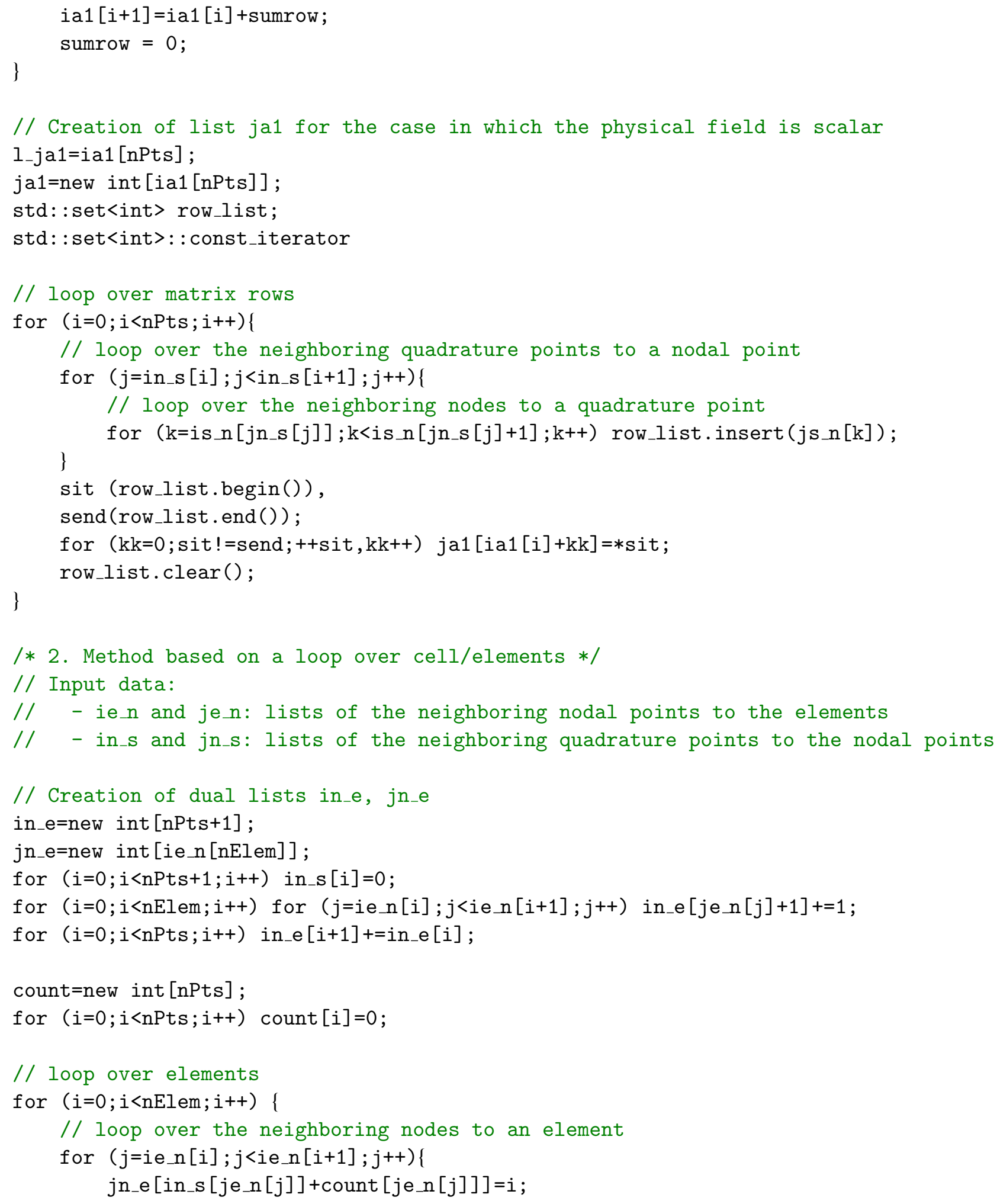




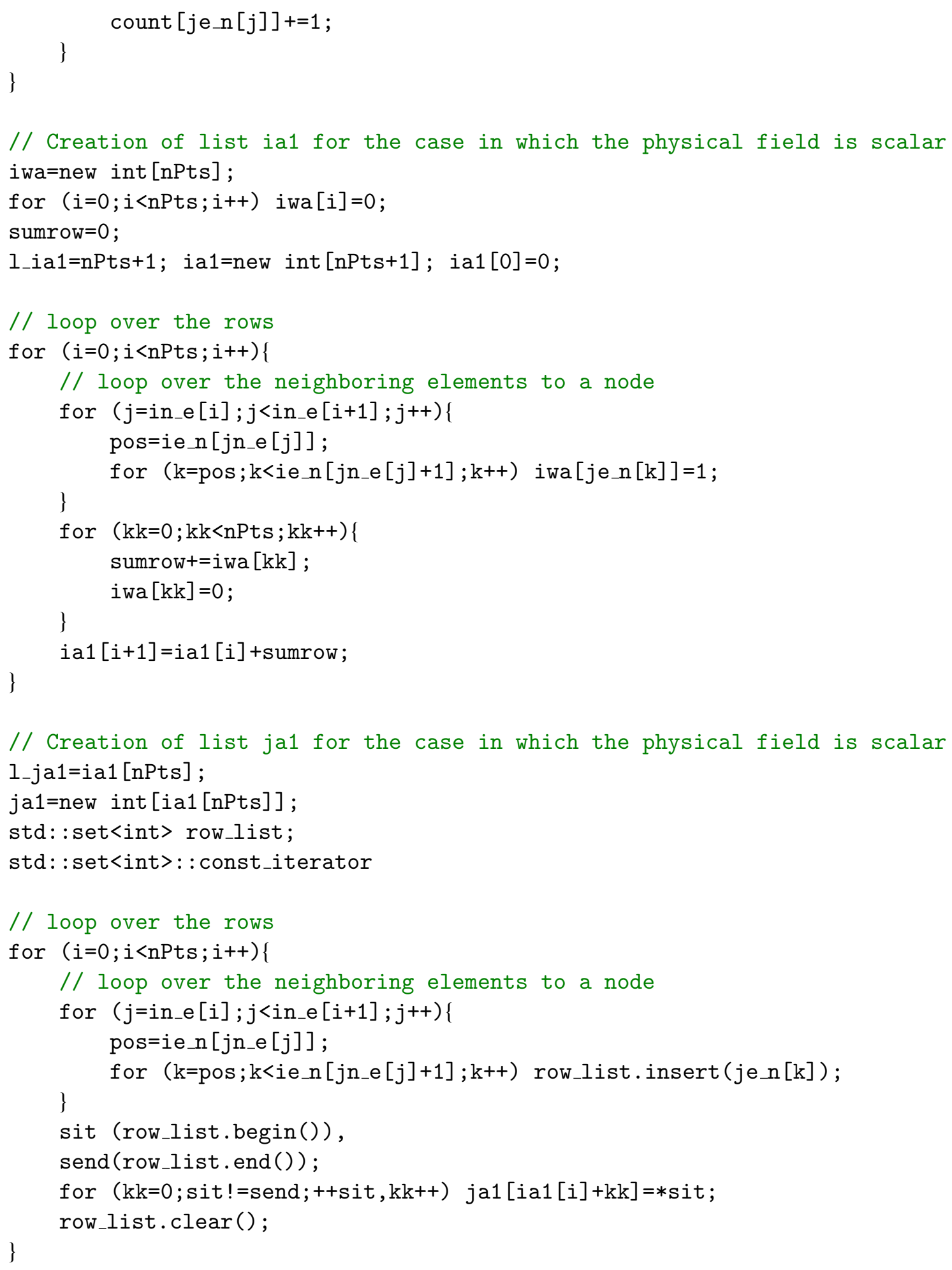




\section{Appendix C.2.2. CreateStructureND()}

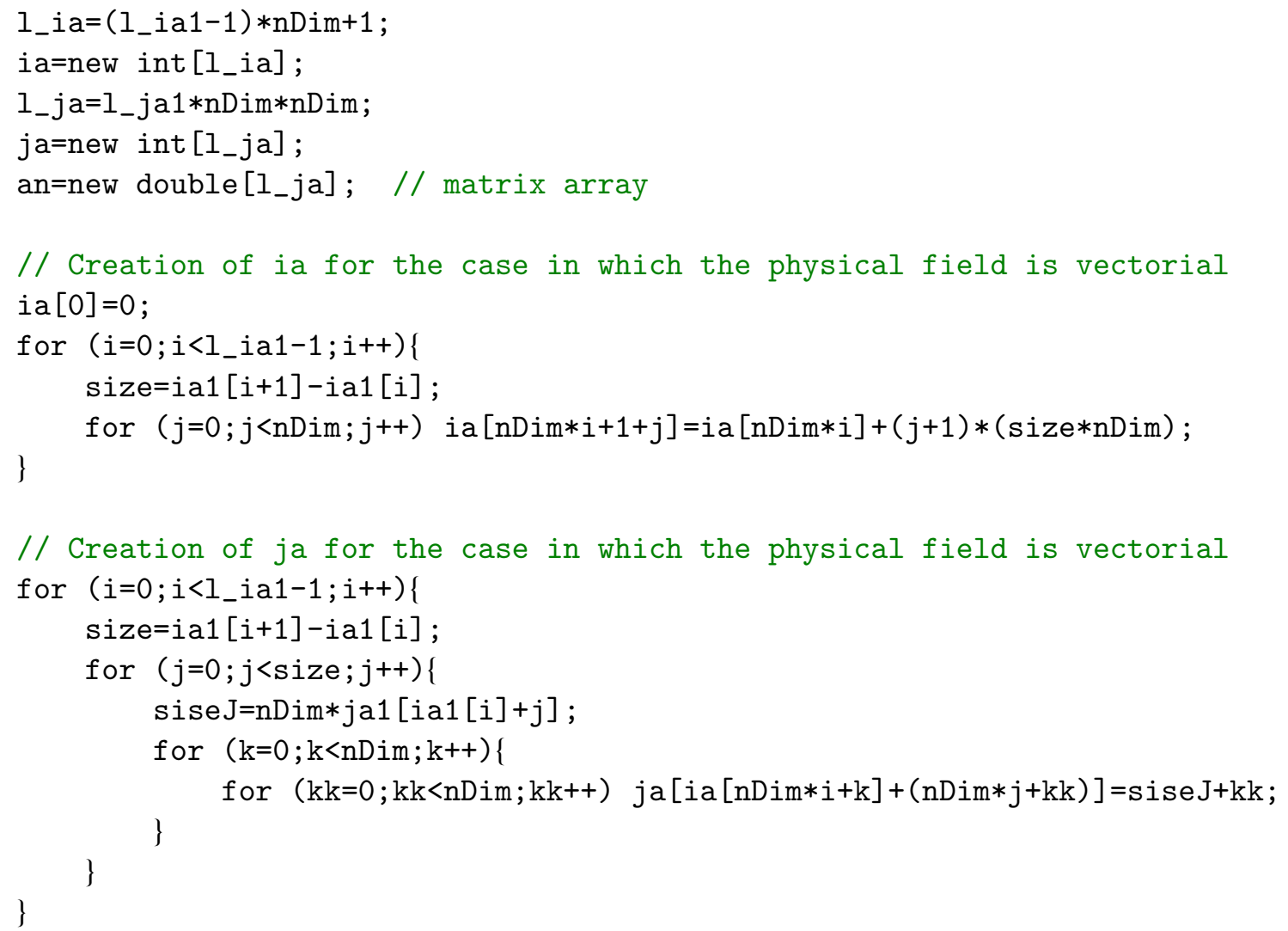

\section{Appendix C.2.3. FillStructureND()}

/* 1. Method based on a loop over the quadrature points */

M=new double[nDim*nNNMax*nDim*nNNMax]; // quadrature point local matrix

for $(k=0 ; k<s P t s ; k++)\{/ /$ loop over quadrature points (sPts is the number of Gauss points) size=is_n $[\mathrm{k}+1]-\mathrm{is} \_\mathrm{n}[\mathrm{k}]$;

for $(i=0 ; i<n D i m * n N N M a x * n D i m * n N N M a x ; i++) \quad M[i]=0.0$;

for $(i=0 ; i<$ size; $i++)\{/ /$ loop over neighbors

for $(j=0 ; j<$ size $j++)\{/ /$ loop over neighbors

for (ii=0;ii<nDim*nDim;ii++) $A[i i]=0.0$;

(*pfunction)(A,parameters,shape_functions); // operation --> get matrix A

// fill local matrix $M$

for ( $i i=0 ; i i<n D i m ; i i++)$

for $(j j=0 ; j j<n D i m ; j j++)$

$M[(n D i m * i+i i) *(\operatorname{size} * n D i m)+(n D i m * j)+j j]=A[i i * n D i m+j j]$;

\} 


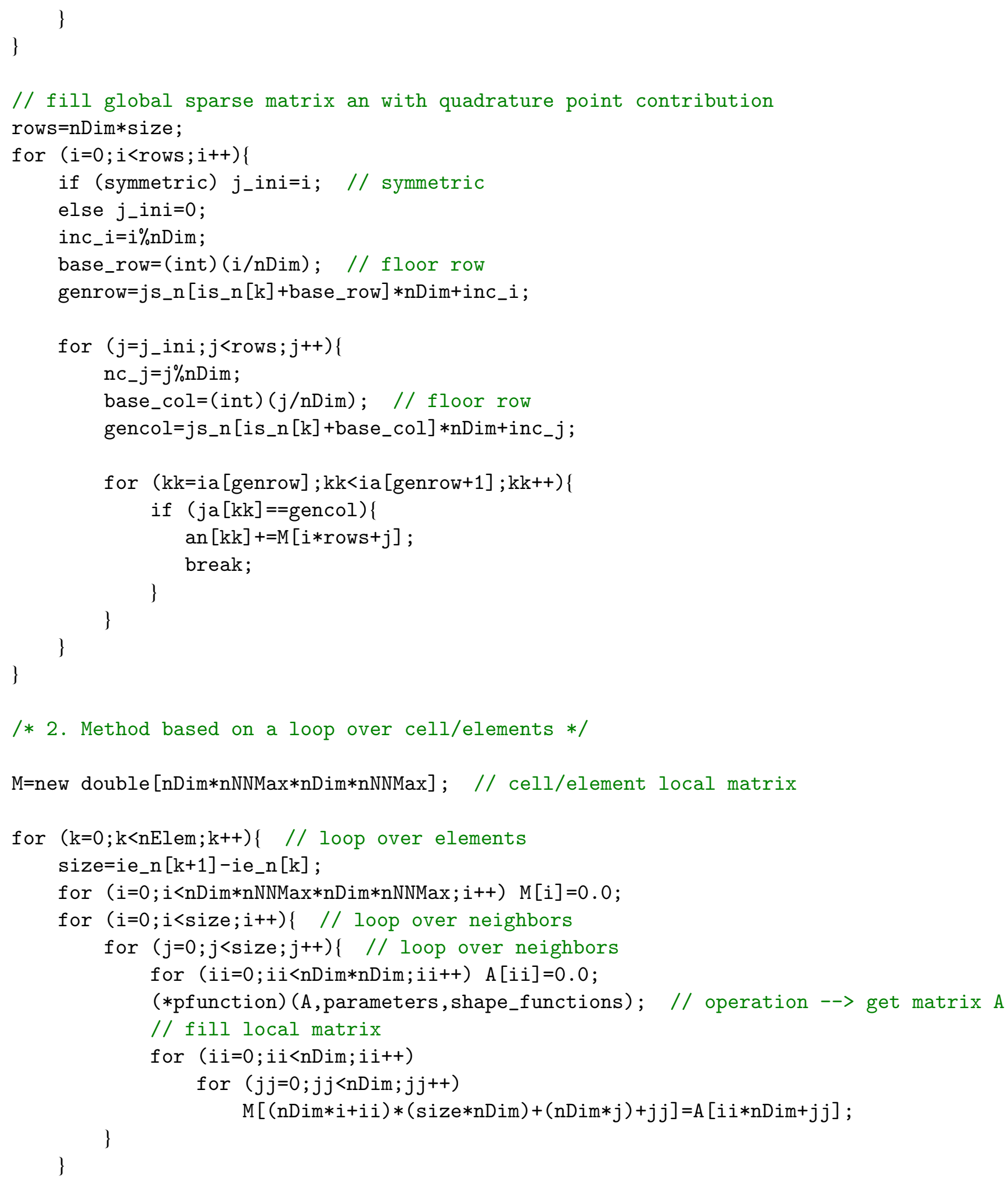




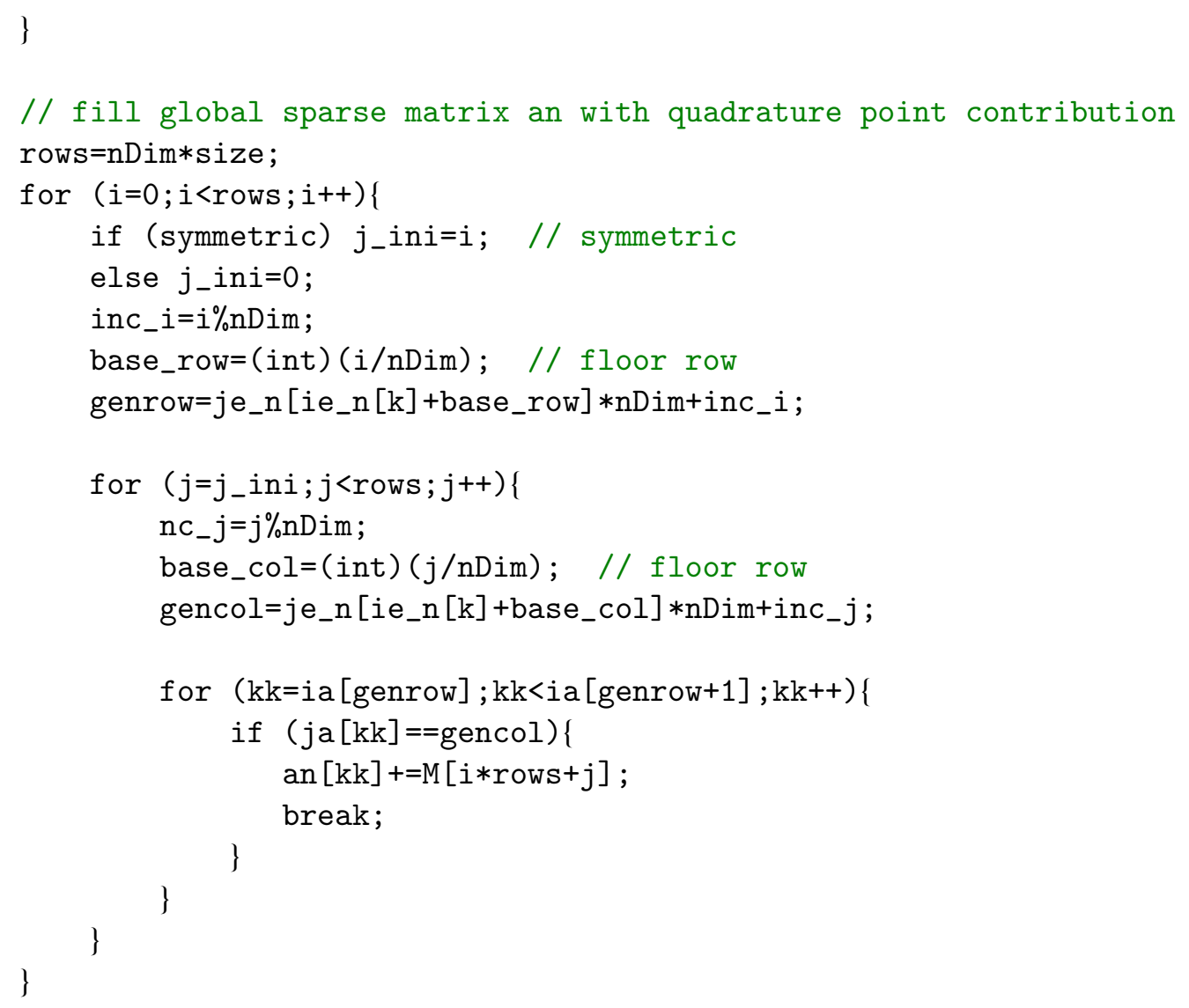

\section{References}

1. Belytschko, T., Krongauz, Y., Organ, D., Fleming, M., Krysl, P.. Meshless methods: An overview and recent developments. Computer Methods in Applied Mechanics and Engineering 1996;139(1):3-47.

2. Li, S., Liu, W.K.. Meshfree and particle methods and their applications. Applied Mechanics Reviews 2002;55(1):1-34.

3. Fries, T., Matthies, H.. Classification and overview of meshfree methods. Tech. Rep.; Institute of Scientific Computing, Technical University Braunschweig, Germany; July 2004.

4. Huerta, A., Belytschko, T., Fernández-Méndez, S., Rabczuk, T.. Meshfree Methods; vol. 1 of Encyclopedia of Computational Mechanics. E. Stein and R. de Borst and T.J.R. Hughes (eds.); chap. 10. John Wiley \& Sons, Ltd.; 2004, p. 279-309.

5. Fasshauer, G.E.. Meshfree Methods; chap. 2. Handbook of Theoretical and Computational Nanotechnology. M. Rieth and W. Schommers (eds.). American Scientific Publishers; 2006, p. 33-97. 
6. Nguyen, V.P., Rabczuk, T., Bordas, S., Duflot, M.. Meshless methods: A review and computer implementation aspects. Mathematics and Computers in Simulation 2008; 79(3):763-813.

7. Chen, J.S., Pan, C., Wu, C.T., Liu, W.K.. Reproducing kernel particle methods for large deformation analysis of nonlinear structures. Computer Methods in Applied Mechanics and Engineering 1996;139:195-227.

8. Chen, J.S., Pan, C., Rogue, C.M.O.L., Wang, H.P.. A lagrangian reproducing kernel particle method for metal forming analysis. Computational Mechanics 1998;22:289-307.

9. Li, B., Habbal, F., Ortiz, M.. Optimal transportation meshfree approximation schemes for fluid and plastic flows. International Journal for Numerical Methods in Engineering 2010;83(12):1541-1579.

10. Combe, U.H., Korn, C.. An adaptive approach with the element-free-galerkin method. Computer Methods in Applied Mechanics and Engineering 1998;162:203-222.

11. Duarte, C.A., Oden, J.T.. An h-p adaptive method using clouds. Computer Methods in Applied Mechanics and Engineering 1996;139:237-262.

12. Dolbow, J., Belytschko, T.. Numerical integration of the galerkin weak form in meshfree methods. Computational Mechanics 1999;23:219-230.

13. Babuška, I., Banerjee, U., Osborn, J.E., Li, Q.. Quadrature for meshless methods. International Journal for Numerical Methods in Engineering 2008;76:1434-1470.

14. Fernández-Méndez, S., Huerta, A.. Imposing essential boundary conditions in meshfree methods. Computer Methods in Applied Mechanics and Engineering 2004;193(1214):1257-1275.

15. Monaghan, J.. An introduction to SPH. Computer Physics Communications 1988;48:8996.

16. Liu, W., Jun, S., Zhang, Y.. Reproducing kernel particle methods. International Journal for Numerical Methods in Fluids 1995;20:1081-1106.

17. Melenk, J.M., Babuška, I.. The partition of unity finite element method : Basic theory and applications. Computer Methods in Applied Mechanics and Engineering 1996;139(14):289-314.

18. Belytschko, T., Lu, Y.Y., Gu, L.. Element free Galerkin methods. International Journal for Numerical Methods in Engineering 1994;37:229-256. 
19. De, S., Bathe, K.J.. The method of finite spheres. Computational Mechanics 2000; 25:329-345.

20. Hong, J.W., Bathe, K.J.. Coupling and enrichment schemes for finite element and finite sphere discretizations. Computers and Structures 2005;83:1386-1395.

21. Sukumar, N.. Construction of polygonal interpolants: a maximum entropy approach. International Journal for Numerical Methods in Engineering 2004;61(12):2159-2181.

22. Arroyo, M., Ortiz, M.. Local maximum-entropy approximation schemes: a seamless bridge between finite elements and meshfree methods. International Journal for Numerical Methods in Engineering 2006;65(13):2167-2202.

23. Sukumar, N., Malsch, E.A.. Recent advances in the construction of polygonal finite element interpolants. Archives of Computational Methods in Engineering 2006;13(1):129_ 163.

24. Sukumar, N., Wright, R.W.. Overview and construction of meshfree basis functions: From moving least squares to entropy approximants. International Journal for Numerical Methods in Engineering 2007;70(2):181-205.

25. Rosolen, A., Millán, D., Arroyo, M.. On the optimum support size in meshfree methods: a variational adaptivity approach with maximum entropy approximants. International Journal for Numerical Methods in Engineering 2010;82(7):868-895.

26. Ullah, Z., Coombs, W.M., Augarde, C.E.. An adaptive finite element/meshless coupled method based on local maximum entropy shape functions for linear and nonlinear problems. Computer Methods in Applied Mechanics and Engineering 2013;267:111-132.

27. Hale, J.S., Baiz, P.M.. A locking-free meshfree method for the simulation of sheardeformable plates based on a mixed variational formulation. Computer Methods in Applied Mechanics and Engineering 2012;241-244:311-322.

28. Millán, D., Rosolen, A., Arroyo, M.. Thin shell analysis from scattered points with maximum-entropy approximants. International Journal for Numerical Methods in Engineering 2011;85(6):723-751.

29. Millán, D., Rosolen, A., Arroyo, M.. Nonlinear manifold learning for meshfree finite deformation thin shell analysis. International Journal for Numerical Methods in Engineering 2013;93(7):685-713.

30. Nissen, K., Cyron, C.J., Gravemeier, V., Wall, W.A.. Information-flux method: a meshfree maximum-entropy petrov-galerkin method including stabilised finite element methods. Computer Methods in Applied Mechanics and Engineering 2012;241-244:225237. 
31. Wu, C.T., Young, D.L., Hong, H.K.. Adaptive meshless local maximum-entropy finite element method for convection-diffusion problems. Computational Mechanics 2014; 53:189-200.

32. Rosolen, A., Peco, C., Arroyo, M.. An adaptive meshfree method for phase-field models of biomembranes. Part I: approximation with maximum-entropy approximants. Journal of Computational Physics 2013;249:303-319.

33. Peco, C., Rosolen, A., Arroyo, M.. An adaptive meshfree method for phase-field models of biomembranes. Part II: a Lagrangian approach for membranes in viscous fluids. Journal of Computational Physics 2013;249:320-336.

34. Amiri, F., Anitescu, C., Arroyo, M., Bordas, S.P.A., Rabczuk, T.. XLME interpolants, a seamless bridge between XFEM and enriched meshless methods. Computational Mechanics 2014;53:45-57.

35. Amiri, F., Millán, D., Shen, Y., Rabczuk, T., Arroyo, M.. Phase-field modeling of fracture mechanics in linear thin shells. Theoretical and Applied Fracture Mechanics 2014;69:102-109.

36. Li, B., Peco, C., Millán, D., Arias, I., Arroyo, M.. Phase-field modeling and simulation of fracture in brittle materials with strongly anisotropic surface energy. International Journal for Numerical Methods in Engineering 2014;:DOI: 10.1002/nme.4726.

37. Arroyo, M., Ortiz, M.. Meshfree Methods for Partial Differential Equations III; vol. 57 of Lecture Notes in Computational Science and Engineering; chap. Local Maximum-Entropy Approximation Schemes. Springer; 2007, p. 1-16.

38. Du, Q., Gunzburger, M., Ju, L.. Meshfree, probabilistic determination of point sets and support regions for meshless computing. Computer Methods in Applied Mechanics and Engineering 2002;191(13-14):1349-1366.

39. Bradford Barber, C., Dobkin, D.P., Huhdanpaa, H.. The quickhull algorithm for convex hulls. ACM Transactions on Mathematical Software 1996;22:469-483.

40. Mount, M.D., Arya, S.. A library for approximate nearest neighbor searching. http: //www. cs. umd. edu/ mount/ANN/; 2010.

41. Eijkhout, V.. Distributed sparse data structures for linear algebra operations. Technical Report CS 92-169; Computer Science Department, University of Tennessee; 1992.

42. Saad, Y.. Iterative Methods for Linear Systems. PWS Publishing, Boston; 1996. 
43. Borden, M.J., Hughes, T.J.R., Landis, C.M., Verhoosel, C.V.. A higher-order phasefield model for brittle fracture: Formulation and analysis within the isogeometric analysis framework. Computer Methods in Applied Mechanics and Engineering 2014;273:100118.

44. Balay, S., Brown, J., Buschelman, K., Gropp, W.D., Kaushik, D., Knepley, M.G., et al. Portable, extensible toolkit for scientific computation. http://www.mcs.anl.gov/ petsc; 2013.

45. Karypis, G., Kumar, V.. Metis-ParMetis: Unstructured Graph Partitioning and Sparse Matrix Ordering System, Version 4.0.3. http://www.cs. umn. edu/ metis; 2009. 